\title{
A MURNAGHAN-NAKAYAMA RULE FOR VALUES OF UNIPOTENT CHARACTERS IN CLASSICAL GROUPS
}

\author{
FRANK LÜBECK AND GUNTER MALLE
}

\begin{abstract}
We derive a Murnaghan-Nakayama type formula for the values of unipotent characters of finite classical groups on regular semisimple elements. This relies on Asai's explicit decomposition of Lusztig restriction. We use our formula to show that most complex irreducible characters vanish on some $\ell$-singular element for certain primes $\ell$.

As an application we classify the simple endotrivial modules of the finite quasi-simple classical groups. As a further application we show that for finite simple classical groups and primes $\ell \geq 3$ the first Cartan invariant in the principal $\ell$-block is larger than 2 unless Sylow $\ell$-subgroups are cyclic.
\end{abstract}

\section{INTRODUCTION}

The classical Murnaghan-Nakayama rule provides an efficient recursive method to compute the values of irreducible characters of symmetric groups. This method can be adapted to the finite general linear and unitary groups, where it then allows us to compute values of unipotent characters not on arbitrary but just on regular semisimple elements; see [10, Prop. 3.3]. This adaptation uses the fact that the unipotent characters of general linear groups coincide with Lusztig's so-called almost characters and then applies the Murnaghan-Nakayama rule for the symmetric group.

In the present paper, we derive a Murnaghan-Nakayama rule for the values of unipotent characters of the finite classical groups on regular semisimple elements; see Theorem 3.3. Our approach here is not via a corresponding formula for the underlying Weyl group (of type $B_{n}$ or $D_{n}$ ), since for classical groups Lusztig's Fourier transform is non-trivial and thus the relation between almost characters and unipotent characters becomes quite tricky. Instead we combine Asai's result expressing the decomposition of Lusztig induction in terms of hooks and cohooks of symbols and which already has a Murnaghan-Nakayama-like form, with Lusztig's character formula.

As a first application we derive a vanishing result for irreducible characters of quasi-simple groups on $\ell$-singular elements:

Theorem 1. Let $\ell>2$ be a prime and $G$ a finite quasi-simple group of $\ell$-rank at least 3. Then for any non-trivial character $\chi \in \operatorname{Irr}(G)$ there exists an $\ell$-singular element $g \in G$ with $\chi(g)=0$, unless either $G$ is of Lie type in characteristic $\ell$, or $\ell=5$ and one of:

Received by the editors August 11, 2015 and, in revised form, January 11, 2016.

2010 Mathematics Subject Classification. Primary 20C20; Secondary 20C33, 20C34.

Key words and phrases. Murnaghan-Nakayama rule, classical groups, simple endotrivial modules, Loewy length, zeroes of characters.

The second author gratefully acknowledges financial support by ERC Advanced Grant 291512. 
(1) $G=\mathrm{L}_{5}(q)$ with $5 \|(q-1)$ and $\chi$ is unipotent of degree $\chi(1)=q^{2} \Phi_{5}$;

(2) $G=\mathrm{U}_{5}(q)$ with $5 \|(q+1)$ and $\chi$ is unipotent of degree $\chi(1)=q^{2} \Phi_{10}$;

(3) $G=L y$ and $\chi(1) \in\{48174,11834746\}$; or

(4) $G=E_{8}(q)$ with $q$ odd, $d_{\ell}(q)=4$ and $\chi$ one character in the Lusztig-series of type $D_{8}$.

The second main ingredient in the proof, apart from our Murnaghan-Nakayama formula, is a result asserting the existence of $\ell$-singular regular semisimple elements in classical groups of Lie type (see Lemma 2.1) which may be of independent interest.

We then apply this vanishing result in order to classify simple endotrivial modules over fields $k$ of positive characteristic $\ell$ for finite classical groups.

Theorem 2. Let $G$ be a finite quasi-simple group of classical Lie type $B_{n}, C_{n}, D_{n}$ or ${ }^{2} D_{n}$ with non-cyclic Sylow $\ell$-subgroup. Then there exists a non-trivial simple endotrivial $k G$-module $V$ if and only if $G=\operatorname{Sp}_{8}(2), k$ has characteristic $\ell=5$, and $\operatorname{dim}(V)=51$.

Our approach relies on the fact, proven in [11, Thm. 1.3] that any endotrivial module is liftable to a characteristic 0 representation, which can then be studied by ordinary character theory. In particular, its character cannot vanish on any $\ell$-singular element, and this latter condition can be checked with the previous Murnaghan-Nakayama formula.

As a further application we give a (partial) answer to a question of Koshitani, Külshammer and Sambale [9, which is then used in [10] to settle this question completely:

Theorem 3. Let $G$ be a finite simple group of classical Lie type $B_{n}, C_{n}, D_{n}$ or ${ }^{2} D_{n}$. Let $\ell>2$ be a prime for which Sylow $\ell$-subgroups of $G$ are non-cyclic. Then the $\ell$-modular projective cover of the trivial character of $G$ has at least three ordinary constituents.

In particular, the first Cartan invariant of $G$ satisfies $c_{11} \geq 3$.

Our paper is organised as follows. In Section 2 we prove the existence of regular semisimple elements in suitable maximal tori of groups of classical type and determine possible overgroups of collections of maximal tori. Section 3 contains the proof of the Murnaghan-Nakayama rule; see Theorem 3.3. We apply this in Section 4 to prove a vanishing result on $\ell$-singular elements; see Theorems 4.1 and 4.2 . In Section [5] we show Theorem 2 classifying simple endotrivial modules for classical groups. In Section 6 we collect the previous results to prove the vanishing result in Theorem 1. Finally, in Section 7.1 we show the application to the proof of Theorem 3 .

\section{MAXimal TORI IN ClASSiCAL GROUPS}

In this section we establish the existence of regular semisimple $\ell$-singular elements in suitable tori of classical groups of Lie type. This will be a main ingredient in the proof of the vanishing Theorem[1. Moreover, we formulate a criterion for collections of maximal tori of simple algebraic groups not to be contained in a common proper reductive subgroup. 


\subsection{Weyl groups and maximal tori in symplectic and orthogonal groups.} For a prime power $q$ and $K=\overline{\mathbb{F}_{q}}$ we consider the natural representation of $\mathbf{G}=$ $\mathrm{Sp}_{2 n}(K)$ on $V=K^{2 n}$ (case $C_{n}$ ) and of $\mathbf{G}=\mathrm{SO}_{2 n+1}(K)$ on $V=K^{2 n+1}$, and a Steinberg morphism $F$ on $\mathbf{G}$ with fixed point group $\mathbf{G}^{F}=\operatorname{Sp}_{2 n}(q)$, respectively, $\mathbf{G}^{F}=\mathrm{SO}_{2 n+1}(q)$. With respect to an appropriate choice of basis of $V$ we can assume (see [13, Exmp. 6.7]) that the subgroup $\mathbf{T}$ of diagonal matrices forms an $F$ stable maximal torus of $\mathbf{G}$, its elements have the form $\operatorname{diag}\left(t_{1}, \ldots, t_{n}, t_{n}^{-1}, \ldots, t_{1}^{-1}\right)$, respectively, $\operatorname{diag}\left(t_{1}, \ldots, t_{n}, 1, t_{n}^{-1}, \ldots, t_{1}^{-1}\right)$, and $F$ acts by raising the diagonal entries to their $q$-th power. The Weyl group $W$ acts on $\mathbf{T}$ by its natural permutation action on the diagonal entries. More precisely, $W$ is isomorphic to the wreath product $C_{2} \prec \mathfrak{S}_{n}$, where the cyclic groups of order 2 interchange the entries $\left\{t_{i}, t_{i}^{-1}\right\}, i=1, \ldots, n$, while the symmetric group permutes the $n$ pairs $\left\{t_{i}, t_{i}^{-1}\right\}$. See [5, Chap. 15] for more details.

The $\mathbf{G}^{F}$-conjugacy classes of $F$-stable maximal tori of $\mathbf{G}$ are parameterised by the conjugacy classes of $W$. An element $t \in \mathbf{T}$ is conjugate to an element $t^{\prime} \in \mathbf{G}^{F}$ if ${ }^{w} F(t)=t$ for some $w \in W$. Then $t^{\prime}$ lies in an $F$-stable maximal torus parameterised by the class of $w$. Such an element $t^{\prime}$ is regular semisimple if $w$ is unique.

The conjugacy classes of $W$ are parameterised by pairs of partitions $(\lambda, \mu) \vdash n$ of $n$ as follows: If $w \in W$ permutes entries of a regular diagonal element as above, then a cycle containing entries $t_{i}$ and $t_{i}^{-1}$ for some $i$ is of even length $2 \mu_{j}$ and contributes a part $\mu_{j}$ to $\mu$. Otherwise a cycle of length $\lambda_{j}$ permutes some diagonal entries and there is another cycle of the same length permuting the inverse diagonal entries in the same way; this contributes a part $\lambda_{j}$ to $\lambda$.

We can embed an even dimensional orthogonal group $\mathbf{G}=\mathrm{SO}_{2 n}(K)$ (type $D_{n}$ ) into $\tilde{\mathbf{G}}=\mathrm{SO}_{2 n+1}(K)$ such that it is stable under the Steinberg morphism of $\tilde{\mathbf{G}}$ and its fixed points are $\mathbf{G}^{F}=\mathrm{SO}_{2 n}^{+}(q)$. There is a second Steinberg morphism $F^{\prime}$ on $\mathbf{G}$ leading to the twisted groups $\mathbf{G}^{F^{\prime}}=\mathrm{SO}_{2 n}^{-}(q)$. The Weyl group $W$ of $\mathbf{G}$ is a subgroup of index 2 in the Weyl group $\tilde{W}$ of $\tilde{\mathbf{G}}$, consisting of the conjugacy classes of $\tilde{W}$ whose parameter $(\lambda, \mu)$ has an even number of parts in $\mu$. If $\mu$ is empty in $(\lambda, \mu)$ and $\lambda$ has only even parts then the corresponding class in $\tilde{W}$ splits into two classes in $W$, called degenerate classes. The twisted Steinberg morphism $F^{\prime}$ acts on $W$ like conjugation by the short root generator of $\tilde{W}$. This way the $F^{\prime}$-conjugacy classes of $W$ can be parameterised by the conjugacy classes of $\tilde{W}$ whose parameter $(\lambda, \mu)$ has an odd number of parts in $\mu$. Since $\mathbf{G}$ and $\tilde{\mathbf{G}}$ have the same rank, maximal tori of $\mathbf{G}$ are also maximal tori of $\tilde{\mathbf{G}}$.

Let $(\lambda, \mu)=\left(\left(\lambda_{1}, \ldots, \lambda_{r}\right),\left(\mu_{1}, \ldots, \mu_{s}\right)\right) \vdash n$. Then the order of the corresponding maximal torus $\mathbf{T}^{F}$ in $\mathbf{G}^{F}$ is

$$
\left|\mathbf{T}^{F}\right|=\prod_{i=1}^{r}\left(q^{\lambda_{i}}-1\right) \prod_{i=1}^{s}\left(q^{\mu_{i}}+1\right)
$$

and $\mathbf{T}^{F}$ contains cyclic subgroups of orders $q^{\lambda_{i}}-1$ and $q^{\mu_{i}}+1$ for all $i$.

2.2. Regular semisimple elements. We need to know that certain maximal tori contain regular elements. As before, let $q$ be a prime power. For a prime $\ell$ not dividing $q$ we denote by $d_{\ell}(q)$ the order of $q$ modulo $\ell$, that is, the smallest $d \geq 1$ with $\ell \mid\left(q^{d}-1\right)$. If $d$ is even then we have $\ell \mid\left(q^{d / 2}+1\right)$. With these definitions, $\ell \mid\left(q^{k}-1\right)$ if and only if $d \mid k$, and for even $d$ we have $\ell \mid\left(q^{k}+1\right)$ if and only if $k$ is an odd multiple of $d / 2$. 
Lemma 2.1. Let $\mathbf{G}$ be a simple simply-connected classical group of type $B_{n}, C_{n}$ or $D_{n}$ defined over the finite field $\mathbb{F}_{q}$ with corresponding Steinberg morphism $F$.

Let $(\lambda, \mu)=\left(\left(\lambda_{1}, \ldots, \lambda_{r}\right),\left(\mu_{1}, \ldots, \mu_{s}\right)\right)$ be a pair of partitions of $n$, and $\mathbf{T}$ a corresponding $F$-stable maximal torus of $\mathbf{G}$. Then $\mathbf{T}^{F}$ contains regular elements if one of the following conditions is fulfilled:

(1) $q>3, \lambda_{1}<\lambda_{2}<\ldots<\lambda_{r}$ and $\mu_{1}<\mu_{2}<\ldots<\mu_{s}$;

(2) $q \in\{2,3\}, \lambda_{1}<\lambda_{2}<\ldots<\lambda_{r}, \mu_{1}<\mu_{2}<\ldots<\mu_{s}$, all $\lambda_{i} \neq 2$, and if $\mathbf{G}$ is of type $B_{n}$ or $C_{n}$, then also all $\lambda_{i} \neq 1$; or

(3) $\mathbf{G}$ is of type $D_{n}, 2<\lambda_{1}<\lambda_{2}<\ldots<\lambda_{r}$ and $1=\mu_{1}=\mu_{2}<\mu_{3}<\ldots<\mu_{s}$.

Proof. (a) We use the natural representations of $\tilde{\mathbf{G}}=\operatorname{Sp}_{2 n}(K)$ and $\tilde{\mathbf{G}}=\mathrm{SO}_{2 n+1}(K)$ with the natural Steinberg morphism that raises matrix entries to their $q$-th power, and we consider $\mathrm{SO}_{2 n}^{ \pm}(q)$ as subgroups of $\mathrm{SO}_{2 n+1}(K)$.

We have described the maximal torus $\mathbf{T}$ of diagonal elements in $\tilde{\mathbf{G}}$ in Section 2.1. The connected centraliser $C_{\tilde{\mathbf{G}}}^{\circ}(t)$ of $t \in \mathbf{T}$ equals $\mathbf{T}$ if and only if $\alpha(t)=1$ for all roots $\alpha$ of $\mathbf{G}$ with respect to $\mathbf{T}$. These roots are explicitly given in [5, Chap. 15] (for types $C_{n}$ and $D_{n}$, but type $B_{n}$ is very similar): It is enough to describe a set of positive roots. Their values on $t=\operatorname{diag}\left(t_{1}, t_{2}, \ldots, t_{n}, t_{n}^{-1}, \ldots, t_{1}^{-1}\right)$, respectively, $t=\operatorname{diag}\left(t_{1}, t_{2}, \ldots, t_{n}, 1, t_{n}^{-1}, \ldots, t_{1}^{-1}\right)$ are:

(Types $\left.B_{n}, C_{n}, D_{n}\right) \alpha(t)=t_{i} t_{j}^{-1}$ and $\alpha(t)=t_{i} t_{j}$ for $1 \leq i<j \leq n$,

(Type $B_{n}$ only) $\alpha(t)=t_{i}$ for $1 \leq i \leq n$,

(Type $C_{n}$ only) $\alpha(t)=t_{i}^{2}$ for $1 \leq i \leq n$.

So, $C_{\tilde{\mathbf{G}}}^{\circ}(t)=\mathbf{T}$ if and only if for all $1 \leq i<j \leq n$ we have $t_{i} \neq t_{j}$ and $t_{i} \neq t_{j}^{-1}$, and in type $B_{n}$ we also have $t_{i} \neq 1$ for $1 \leq i \leq n$, and in type $C_{n}$ we also have $t_{i} \neq \pm 1$ for $1 \leq i \leq n$.

(b) In type $C_{n}$ we have $\mathbf{G}=\tilde{\mathbf{G}}$, while in types $B_{n}$ and $D_{n}$ the groups $\mathrm{SO}_{2 n+1}(K)$ and $\mathrm{SO}_{2 n}(K)$ are not simply-connected. But there are isogenies from the simplyconnected coverings $\mathbf{G}=\operatorname{Spin}_{m}(K) \rightarrow \mathrm{SO}_{m}(K)=\tilde{\mathbf{G}}$. If $q$ is even, that is, $\operatorname{char}(K)=2$, then this map has a trivial kernel and induces isomorphisms $\operatorname{Spin}_{m}^{ \pm}(q) \rightarrow \mathrm{SO}_{m}^{ \pm}(q)$. For odd $q$ the kernel is a central subgroup of order 2, and the image of the induced map $\operatorname{Spin}_{m}^{ \pm}(q) \rightarrow \mathrm{SO}_{m}^{ \pm}(q)$ of the finite groups has index 2 .

To show the existence of regular elements in tori of type $(\lambda, \mu)$ we construct a $t \in \mathbf{T}$ such that ${ }^{w} F(t)=t$ for some $w \in W$ in the class $(\lambda, \mu)$ and $C_{\tilde{\mathbf{G}}}^{\circ}(t)=\mathbf{T}$. In cases $B_{n}$ and $D_{n}$ and odd $q$ this $t$ will be a square of another element in $\mathbf{T}^{w F}$ such that in all cases $t$ has a preimage in a corresponding twisted maximal torus of the simply-connected group $\mathbf{G}$. This preimage is regular because centralisers of semisimple elements in a simply-connected group are connected.

(c) Now let $(\lambda, \mu)=\left(\left(\lambda_{1}, \ldots, \lambda_{r}\right),\left(\mu_{1}, \ldots, \mu_{s}\right)\right) \vdash n$ be a pair of partitions. We construct $t \in \mathbf{T}$ by describing the entries $t_{1}, \ldots, t_{n} \in K$.

First assume that $\mathbf{G}$ is of type $C_{n}$ or that $q$ is even.

For $1 \leq i \leq r$ let $a_{i} \in \mathbb{F}_{q^{\lambda_{i}}}$ be of order $q^{\lambda_{i}}-1$ and use $a_{i}, a_{i}^{q}, \ldots, a_{i}^{q^{\lambda_{i}-1}}$ as entries of $t$ (note that $a_{i}^{q^{\lambda_{i}}}=a_{i}$ ).

For $1 \leq j \leq s$ let $b_{j} \in \mathbb{F}_{q^{2 \mu_{j}}}$ be of order $q^{\mu_{j}}+1$ and use $b_{j}, b_{j}^{q}, \ldots, b_{j}^{q^{\mu_{j}-1}}$ as entries of $t$ (note that then $b_{j}^{q^{\mu_{j}}}=b_{j}^{-1}$ ).

If $\mathbf{G}$ is of type $B_{n}$ or $D_{n}$ and $q$ is odd, we use as $t$ the square of the element just described (so $a_{i}$ is of order $\frac{1}{2}\left(q^{\lambda_{i}}-1\right)$ and $b_{j}$ is of order $\frac{1}{2}\left(q^{\mu_{j}}+1\right)$ ). 
It is clear from this construction that the Steinberg morphism $F$ of $\operatorname{Sp}_{2 n}(K)$, respectively, $\mathrm{SO}_{2 n+1}(K)$ permutes the entries of $t$ such that this can be reversed by an element $w \in W$ of cycle type $(\lambda, \mu)$, so $t={ }^{w} F(t)$.

(d) We next discuss when $t$ fails to be regular. Let $\lambda_{i}, \mu_{j}>0$ with $q^{\lambda_{i}}-1=$ $q^{\mu_{j}}+1$. Then $\mu_{j}<\lambda_{i}$ and the assumption is equivalent to $q^{\mu_{j}}\left(q^{\lambda_{i}-\mu_{j}}-1\right)=2$. It follows that $q=2, \mu_{j}=1$ and $\lambda_{i}=2$.

(e) Let $k \in \mathbb{Z}, 0<k<\lambda_{i}$ and assume $\left(q^{\lambda_{i}}-1\right) \mid\left(q^{k} \mp 1\right)$. Then $q^{\lambda_{i}}-1 \leq q^{k} \mp 1$, which holds if and only if $q^{k}\left(q^{\lambda_{i}-k}-1\right) \leq 0$ or 2 , respectively. This is only possible for $q=2, k=1$ and $\lambda_{i}=2$. Similarly, for odd $q$ the condition $\frac{1}{2}\left(q^{\lambda_{i}}-1\right) \mid\left(q^{k} \mp 1\right)$ implies $q=3, k=1$ and $\lambda_{i}=2$.

Now let $k \in \mathbb{Z}, 0<k<\mu_{j}$. A similar argument as above shows that for all $q$ we have $\left(q^{\mu_{j}}+1\right) \nmid\left(q^{k} \mp 1\right)$ and for odd $q$ we also have $\frac{1}{2}\left(q^{\mu_{j}}+1\right) \nmid\left(q^{k} \mp 1\right)$.

(f) Now we prove assertions (1) and (2) of the statement by showing that in those cases the element $t \in \mathbf{T}$ constructed in (c) does not lie in the kernel of any root.

First note that the $a_{i}(1 \leq i \leq r)$ and $b_{j}(1 \leq j \leq s)$ have pairwise different orders. This follows from (d) since for $q=2$ we assume that all $\lambda_{i} \neq 2$.

According to (a) we need to show that for $1 \leq i \leq r$ any of the entries $\left(a_{i}, a_{i}^{q}, \ldots, a_{i}^{q^{\lambda_{i}-1}}\right)$ are not equal or inverse to each other, and similarly for the $\left(b_{j}, b_{j}^{q}, \ldots, b_{j}^{q^{\mu_{j}-1}}\right)$. Since the map $x \mapsto x^{q}$ permutes these entries of $t$ and their inverses it suffices to show that for $0<k<\lambda_{i}$ we have $a_{i}^{q^{k}} \neq a_{i}^{ \pm 1}$ (equivalently $\left.a_{i}^{q^{k} \mp 1} \neq 1\right)$, and similarly for the $b_{j}$. Now $a_{i}^{q^{k} \mp 1}=1$ implies $\left(q^{\lambda_{i}}-1\right) \mid\left(q^{k} \mp 1\right)$ or $\frac{1}{2}\left(q^{\lambda_{i}}-1\right) \mid\left(q^{k} \mp 1\right)$, respectively. Using (e) this implies $\lambda_{i}=2$ and $q \in\{2,3\}$, contradicting the assumptions.

Similarly, step (e) shows that we always have $b_{j}^{q^{k}} \neq b_{j}^{ \pm 1}$ for $0<k<\mu_{j}$.

For type $C_{n}$ we also have to show that $a_{i}^{2} \neq 1(1 \leq i \leq r)$ and $b_{j}^{2} \neq 1(1 \leq j \leq s)$. This holds because $a_{i}^{2}=1$, i.e., $\left(q^{\lambda_{i}}-1\right) \mid 2$ implies $\lambda_{i}=1$ and $q \in\{2,3\}$, contradicting the assumption. Note that always $q^{\mu_{i}}+1>2$.

For type $B_{n}$ we also have to show that $a_{i} \neq 1(1 \leq i \leq r)$ and $b_{j} \neq 1(1 \leq j \leq s)$. This follows as $q^{\lambda_{i}}-1=1$ implies $q=2$ and $\lambda_{i}=1$, while $\frac{1}{2}\left(q^{\lambda_{i}}-1\right)=1$ implies $q=3$ and $\lambda_{i}=1$ for odd $q$, and as always $q^{\mu_{j}}+1>1$.

(g) The argument for statement (3) is very similar. A suitable element $t$ can be defined as before, except that for $\mu_{1}=1$ we choose $b_{1}=1$. Then the entry for $\mu_{2}=1$ has order $q+1$ for even $q$ or $\frac{1}{2}(q+1)$ for odd $q$ which is always larger than 1 .

Remark 2.2. Let $\mathbf{G}$ be a connected reductive group with Steinberg morphism $F$ and $\mathbf{T}$ an $F$-stable maximal torus of $\mathbf{G}$. Let $\ell$ be a prime dividing $\left|\mathbf{T}^{F}\right|$. If $\mathbf{T}^{F}$ contains a regular element then $\mathbf{T}^{F}$ also contains an $\ell$-singular regular element.

Proof. Let $t \in \mathbf{T}^{F}$ be an $\ell$-regular (that is of order prime to $\ell$ ) regular element and $u \in \mathbf{T}^{F}$ be any element of order $\ell$. Then $t u$ has order $\ell|t|$ and some power of $t u$ equals $t$, hence $t u$ is also regular.

2.3. Maximal connected reductive subgroups. The following lemma will allow us to decide if a collection of classes of maximal tori of a classical group has representatives inside the centraliser of a semisimple element: 
Lemma 2.3. Let $\mathbf{G}$ be a simple algebraic group of classical type $B_{n}, C_{n}$ (with $n \geq 2$ ) or $D_{n}$ (with $n \geq 4$ ) with Frobenius endomorphism $F$ such that $\mathbf{G}^{F}$ is a classical group. Let $\Lambda$ be a set of pairs of partitions $(\lambda, \mu) \vdash n$. Assume the following:

(1) there is no $1 \leq k \leq n-1$ such that all $(\lambda, \mu) \in \Lambda$ are of the form $\left(\lambda_{1}, \mu_{1}\right) \sqcup$ $\left(\lambda_{2}, \mu_{2}\right)$ (that is, $\lambda$ contains the parts from $\lambda_{1}$ and $\lambda_{2}$ and similarly for $\mu$ ) with $\left(\lambda_{1}, \mu_{1}\right) \vdash k$;

(2) the greatest common divisor of all parts of all $(\lambda, \mu) \in \Lambda$ is 1 ; and

(3) if $\mathbf{G}$ is of type $B_{n}$, then there exist pairs $(\lambda, \mu) \in \Lambda$ for which $\mu$ has an odd number of parts, and for which $\mu$ has an even number of parts.

If $s \in \mathbf{G}^{F}$ is semisimple such that $C_{\mathbf{G}}(s)$ contains maximal tori of $\mathbf{G}$ corresponding to all $(\lambda, \mu) \in \Lambda$ then $s$ is central.

Proof. The types of maximal tori are insensitive to isogeny types, so we may assume that $\mathbf{G}$ is a classical matrix group $\operatorname{Sp}(V)$ or $\mathrm{SO}(V)$. Let $s \in \mathbf{G}^{F}$ be a non-central semisimple element. Then $\mathbf{H}:=C_{\mathbf{G}}^{\circ}(s)$ fixes each eigenspace of $s$ on $V$. Since $s$ is non-central it has at least two different eigenvalues. Since $s$ is $F$-stable, the eigenspaces of $s$ are permuted under the action of $F$. First assume that the set of eigenspaces forms one orbit, of length $f>1$, say, under the action of $F$. Then $\mathbf{H}$ is a central product of isomorphic classical groups, $\mathbf{H}^{F}$ is an extension field subgroup and hence its maximal tori have parameters all parts of which are divisible by $f$, contradicting (2).

So there are at least two $F$-orbits of eigenspaces. Let $V_{1}<V$ be an $F$-stable sum of eigenspaces of minimal possible dimension. Then either $V_{1} \cap V_{1}^{\perp} \neq 0$ or $V_{1}$ is non-degenerate and $V=V_{1} \perp V_{1}^{\perp}$. The stabilisers of totally singular spaces are contained in maximal parabolic subgroups [13. Prop. 12.13], but these only have tori of types as excluded by (1). Else, $\mathbf{H}$ is contained in the stabiliser of $V_{1} \perp V_{1}^{\perp}$, a central product of classical groups. The latter are of types $C_{a}+C_{b}$, respectively, $B_{a}+D_{b}$ or $D_{a}+D_{b}$ with $a+b=n, a, b>0$ for $\mathbf{G}$ of type $C_{n}$, respectively, $B_{n}, D_{n}$. Then the parameters of $F$-stable maximal tori of $\mathbf{H}$ are of the form excluded by (1) of the lemma, for $k=a$, unless $\mathbf{G}$ is of type $B_{n}$ and $V_{1}$ is 1-dimensional, when $\mathbf{H}$ could be of type $D_{n}$. In that case, the number of parts in the second entry of the parameters of $F$-stable maximal tori of $\mathbf{H}$ all have a fixed parity (namely even for $\mathbf{H}^{F}$ untwisted, and odd for $\mathbf{H}^{F}$ twisted). This is excluded by (3).

\section{A Murnaghan-Nakayama rule}

In this section we derive a Murnaghan-Nakayama type formula for the values of unipotent characters of classical groups of Lie type on regular semisimple elements. It relies on a result of Asai on the decomposition of Lusztig restriction.

3.1. Lusztig restriction. We consider the following setup: $\mathbf{G}$ is a connected reductive algebraic group over an algebraic closure of the finite field $\mathbb{F}_{p}$, and $F: \mathbf{G} \rightarrow$ $\mathbf{G}$ is a Steinberg endomorphism of $\mathbf{G}$, with finite group of fixed points $\mathbf{G}^{F}$. For an $F$-stable Levi subgroup $\mathbf{L} \leq \mathbf{G}$ of $\mathbf{G}$ Lusztig defined, via certain $\ell$-adic cohomology constructions, induction and restriction functors

$$
R_{\mathbf{L}}^{\mathbf{G}}: \mathbb{Z} \operatorname{Irr}\left(\mathbf{L}^{F}\right) \rightarrow \mathbb{Z} \operatorname{Irr}\left(\mathbf{G}^{F}\right) \quad \text { and } \quad{ }^{*} R_{\mathbf{L}}^{\mathbf{G}}: \mathbb{Z} \operatorname{Irr}\left(\mathbf{G}^{F}\right) \rightarrow \mathbb{Z} \operatorname{Irr}\left(\mathbf{L}^{F}\right)
$$

between the respective groups of virtual characters. These are adjoint to one another with respect to the usual scalar product of characters. We need the following 
connection between Lusztig restriction and ordinary restriction on regular semisimple conjugacy classes:

Proposition 3.1. Let $\mathbf{L} \leq \mathbf{G}$ be an F-stable Levi subgroup. Let $\chi \in \operatorname{Irr}\left(\mathbf{G}^{F}\right)$ and $s \in \mathbf{L}^{F}$ be regular semisimple. Then ${ }^{*} R_{\mathbf{L}}^{\mathbf{G}}(\chi)(s)=\chi(s)$.

Proof. According to the character formula in [5, Prop. 12.2(ii)] we have

$$
{ }^{*} R_{\mathbf{L}}^{\mathbf{G}}(\chi)(s)=\frac{\left|C_{\mathbf{L}}^{\circ}(s)^{F}\right|}{\left|C_{\mathbf{G}}^{\circ}(s)^{F}\right|} \sum_{u \in C_{\mathbf{G}}^{\circ}(s)_{u}^{F}} Q_{C_{\mathbf{L}}^{\circ}(s)}^{C_{\mathbf{G}}^{\circ}(s)}(u, 1) \chi(s),
$$

where the sum runs over unipotent elements in $C_{\mathbf{G}}^{\circ}(s)^{F}$, and $Q_{C_{\mathbf{L}}^{\circ}(s)}^{C^{\circ}(s)}$ is a certain 2-parameter Green function. Since $s$ is assumed to be regular semisimple, $\mathbf{T}:=$ $C_{\mathbf{G}}^{\circ}(s)=C_{\mathbf{L}}^{\circ}(s)$ is a torus. Hence the only term in the sum is the one for $u=1$, and by [5, p. 98] the Green function takes the value

$$
Q_{C_{\mathbf{L}}^{\circ}(s)}^{C_{\mathbf{L}}^{\circ}(s)}(1,1)=Q_{\mathbf{T}}^{\mathbf{T}}(1,1)=1
$$

(again using that $C_{\mathbf{G}}^{\circ}(s)$ contains no non-trivial unipotent elements). The claim follows.

3.2. Symbols. We now specialise to simple groups of classical type. By Lusztig's results their unipotent characters are parameterised by so-called symbols. Here, a symbol is an unordered pair of finite sets of non-negative integers, usually denoted as

$$
\mathcal{S}=\left(\begin{array}{l}
X \\
Y
\end{array}\right)=\left(\begin{array}{llll}
\lambda_{1} & \lambda_{2} & \ldots & \lambda_{r} \\
\mu_{1} & \mu_{2} & \ldots & \mu_{s}
\end{array}\right)
$$

with $\lambda_{1}<\lambda_{2}<\ldots<\lambda_{r}$ and $\mu_{1}<\mu_{2}<\ldots<\mu_{s}$ where $r, s \geq 0$. Two symbols are said to be equivalent if one can be obtained from the other by a sequence of shift operations

$$
\mathcal{S} \mapsto\left(\begin{array}{ccccc}
0 & \lambda_{1}+1 & \lambda_{2}+1 & \ldots & \lambda_{r}+1 \\
0 & \mu_{1}+1 & \mu_{2}+1 & \ldots & \mu_{s}+1
\end{array}\right)
$$

or by interchanging the rows $X$ and $Y$ of $\mathcal{S}$. The rank of $\mathcal{S}$ is by definition

$$
\operatorname{rk}(\mathcal{S})=\sum_{i=1}^{r} \lambda_{i}+\sum_{i=1}^{s} \mu_{i}-\left\lfloor\left(\frac{r+s-1}{2}\right)^{2}\right\rfloor .
$$

The defect of $\mathcal{S}$ is $\operatorname{def}(\mathcal{S}):=|r-s|$. Note that the rank and the defect are welldefined on equivalence classes. We will use the following notation to describe explicit symbols: $\underline{n}:=\{0,1, \ldots, n\}$ and for a set $X$, a $k \in X$ and $l \notin X$ we write $X \backslash k \cup l:=$ $X \backslash\{k\} \cup\{l\}$.

Let $d$ be a positive integer. We say that $\mathcal{S}$ has a $d$-hook $h$ at $x \in X$ if $0 \leq x-d \notin$ $X$, and similarly at $x \in Y$. Removing that $d$-hook leads to the symbol $\mathcal{S} \backslash h:=\left(\begin{array}{c}X^{\prime} \\ Y\end{array}\right)$ with $X^{\prime}=X \backslash x \cup(x-d)$ (respectively $\mathcal{S} \backslash h:=\left(\begin{array}{c}X \\ Y^{\prime}\end{array}\right)$ with $Y^{\prime}=Y \backslash x \cup(x-d)$ ). Attached to the hook $h$ is the sign $\epsilon_{h}:=(-1)^{m}$ where $m:=\mid\{y \in X \mid x-d<$ $y<x\} \mid$ (respectively $m:=|\{y \in Y \mid x-d<y<x\}|$ ). We say that $\mathcal{S}$ has a $d$-cohook $c$ at $x \in X$ if $0 \leq x-d \notin Y$, and similarly at $x \in Y$. Removing that $d$-cohook leads to the symbol $\mathcal{S} \backslash c:=\left(\begin{array}{c}X^{\prime} \\ Y^{\prime}\end{array}\right)$ with $X^{\prime}=X \backslash\{x\}, Y^{\prime}=Y \cup\{x-d\}$ (respectively $Y^{\prime}=Y \backslash\{x\}, X^{\prime}=X \cup\{x-d\}$ ). Attached to the cohook $c$ is the sign $\epsilon_{c}:=(-1)^{m}$, where $m:=|\{y \in X \mid y<x\}|+|\{y \in Y \mid y<x-d\}|$ (respectively $m:=|\{y \in Y \mid y<x\}|+|\{y \in X \mid y<x-d\}|)$; see [6, §3] for example. Note that 
removing a $d$-hook or a $d$-cohook from a symbol of rank $n$ yields a symbol of rank $n-d$.

For a symbol $\mathcal{S}=(X, Y)$ with all entries of $X, Y$ not larger than $m$ we define the dual symbol $\mathcal{S}^{\vee}=\left(X^{\prime}, Y^{\prime}\right)$ where

$$
X^{\prime}=\underline{m} \backslash\{m-x \mid x \in X\} \text { and } Y^{\prime}=\underline{m} \backslash\{m-y \mid y \in Y\} .
$$

The hooks and cohooks of $S$ and $S^{\vee}$ are each in natural bijection such that removing hooks or cohooks commutes with the duality operation. This observation will allow us to simplify some of the later statements and proofs.

3.3. Unipotent characters of symplectic and orthogonal groups. Now assume that $\mathbf{G}$ is simple of type $B_{n}, C_{n}$ or $D_{n}$, and $F$ does not induce the triality automorphism when $\mathbf{G}$ is of type $D_{4}$ nor the exceptional graph automorphism in type $B_{2}$ or $C_{2}$. According to Lusztig [12] the unipotent characters of $G:=\mathbf{G}^{F}$ are then parameterised by (equivalence classes of) symbols of rank $n$. More precisely, if $\mathbf{G}$ is of type $B_{n}$ or $C_{n}$, the unipotent characters of $G$ are parameterised by symbols of rank $n$ and odd defect. Now assume that $\mathbf{G}$ is of type $D_{n}$. If the Steinberg endomorphism $F$ is untwisted, then the unipotent characters of $G$ are parameterised by symbols $\mathcal{S}$ of $\operatorname{rank} n$ and $\operatorname{defect} \operatorname{def}(\mathcal{S}) \equiv 0(\bmod 4)$, where symbols with two equal rows stand for two characters each. These are the so-called degenerate symbols. If $\mathbf{G}^{F}$ is of twisted type, then the unipotent characters of $G$ are parameterised by symbols $\mathcal{S}$ of rank $n$ and $\operatorname{defect} \operatorname{def}(\mathcal{S}) \equiv 2(\bmod 4)$.

If $\mathcal{S}$ is a symbol parameterising a unipotent character $\rho$ of a classical group then the Alvis-Curtis dual $\rho^{\vee}$ of $\rho$ is parameterised by the dual symbol $\mathcal{S}^{\vee}$ which has the same rank, the same defect and the same hook and cohook lengths (see 12, 4.5.5, 4.6.8]). (In fact, the degree $\rho^{\vee}(1)$ differs by a power of the underlying characteristic of $\mathbf{G}$ from the degree $\rho(1)$.)

3.4. Values of unipotent characters at regular semisimple elements. We first recall the following result of Asai on the decomposition of Lusztig induction as given in [6, (3.1), (3.2)]. We restate it in terms of the restriction functors ${ }^{*} R_{\mathbf{L}}^{\mathbf{G}}$ instead of the induction functors $R_{\mathrm{L}}^{\mathbf{G}}$ as in the cited reference. This is a summary of results from [1, 2.8], [2, 1.5] and [3, 2.2.3]. Note that there is a sign missing in the formula [6, (3.2)] in the case $D_{n}$ as can be seen by considering the trivial character.

Theorem 3.2 (Asai (1984)). Let $\mathbf{G}$ be of type $B_{n}, C_{n}$ or $D_{n}$. Let $d \geq 1$. Let $\mathcal{S}$ be a symbol of a unipotent character of $G=\mathbf{G}^{F}$. If $\mathcal{S}$ is not degenerate, we write $\rho_{\mathcal{S}}$ for the corresponding character. If $\mathcal{S}$ is degenerate we write $\rho_{\mathcal{S}}$ for the sum of the two corresponding characters.

(a) Let $\mathbf{L}$ be the centraliser in $\mathbf{G}$ of an F-stable torus $\mathbf{T}_{d}$ of $\mathbf{G}$ with $\left|\mathbf{T}_{d}^{F}\right|=q^{d}-1$. Then

$$
{ }^{*} R_{\mathbf{L}}^{\mathbf{G}}\left(\rho_{\mathcal{S}}\right)=\sum_{h \text { d-hook }} \epsilon_{h} \rho_{\mathcal{S} \backslash h}
$$

where $h$ runs over the d-hooks of $\mathcal{S}$.

(b) Let $\mathbf{L}$ be the centraliser in $\mathbf{G}$ of an F-stable torus $\mathbf{T}_{d}$ of $\mathbf{G}$ with $\left|\mathbf{T}_{d}^{F}\right|=q^{d}+1$. Then

$$
{ }^{*} R_{\mathbf{L}}^{\mathbf{G}}\left(\rho_{\mathcal{S}}\right)=(-1)^{\delta} \sum_{c \text { d-cohook }} \epsilon_{c} \rho_{\mathcal{S} \backslash c}
$$

where $c$ runs over the $d$-cohooks of $\mathcal{S}$, and $\delta=0$ for types $B_{n}, C_{n}, \delta=1$ for type $D_{n}$. 
With this we can show a Murnaghan-Nakayama formula for values of unipotent characters at regular semisimple elements.

Theorem 3.3. Let $\mathbf{G}$ be of type $B_{n}, C_{n}$ or $D_{n}$. Let $\mathcal{S}$ be a symbol of a unipotent character of $G=\mathbf{G}^{F}$. If $\mathcal{S}$ is not degenerate, we write $\rho_{\mathcal{S}}$ for the corresponding character. If $\mathcal{S}$ is degenerate we write $\rho_{\mathcal{S}}$ for the sum of the two corresponding characters. Let $s \in G$ be regular semisimple lying in an $F$-stable maximal torus $\mathbf{T}$ of $\mathbf{G}$ parameterised by $(\lambda, \mu) \vdash n$.

(a) If $\lambda$ has a part of length $d$ then

$$
\rho_{\mathcal{S}}(s)=\sum_{h \text { d-hook }} \epsilon_{h} \rho_{\mathcal{S} \backslash h}(s)
$$

where $h$ runs over the d-hooks of $\mathcal{S}$.

(b) If $\mu$ has a part of length $d$ then

$$
\rho_{\mathcal{S}}(s)=(-1)^{\delta} \sum_{c \text {-cohook }} \epsilon_{c} \rho_{\mathcal{S} \backslash c}(s)
$$

where $c$ runs over the $d$-cohooks of $\mathcal{S}$, and $\delta=0$ for types $B_{n}, C_{n}, \delta=1$ for type $D_{n}$.

On the right-hand side of these formulae the symbols and characters belong to a Levi subgroup of semisimple rank $n-d$ and $s$ lies in a maximal torus of that Levi subgroup corresponding to the pair of partitions $(\lambda \backslash d, \mu) \vdash(n-d)$ in case (a), respectively, $(\lambda, \mu \backslash d) \vdash(n-d)$ in case $(b)$.

Proof. First assume that $\lambda$ has a part of length $d$. Then the torus $\mathbf{T}$ has an $F$-stable subtorus $\mathbf{T}_{d}$ with $\left|\mathbf{T}_{d}^{F}\right|=q^{d}-1$; see Section 2.1 We consider Lusztig restriction of $\rho=\rho_{\mathcal{S}}$ to the $F$-stable Levi subgroup $\mathbf{L}:=C_{\mathbf{G}}\left(\mathbf{T}_{d}\right) \cong \mathbf{H} \mathbf{T}_{d}$, with $\mathbf{H}$ a group of the same type as $\mathbf{G}$ and of rank $n-d$. This is a $d$-split Levi subgroup containing $\mathbf{T}$, so $s$, and according to Asai's Theorem 3.2 (a), the constituents of ${ }^{*} R_{\mathbf{L}}^{\mathbf{G}}(\rho)$ are, up to the $\operatorname{sign} \epsilon_{h}$ as given in the statement, precisely those unipotent characters of $\mathbf{L}^{F}$ whose parameterising symbol is obtained from $\mathcal{S}$ by removing a $d$-hook. Application of Proposition 3.1 then gives the claim in (a).

If $\mu$ has a part of length $d$, then $\mathbf{T}$ has an $F$-stable subtorus $\mathbf{T}_{d}$ with $\left|\mathbf{T}_{d}^{F}\right|=q^{d}+1$, and we can argue precisely as before using Theorem 3.2 (b) for the decomposition of Lusztig restriction to $\mathbf{L}:=C_{\mathbf{G}}\left(\mathbf{T}_{d}\right) \geq \mathbf{T} \ni s$.

Note that in the theorem $\rho_{\mathcal{S} \backslash h}$, respectively, $\rho_{\mathcal{S} \backslash c}$, is a unipotent character of the classical group $\mathbf{L}^{F}$ of rank $n-d$ (of the same type as $G$, except that removing cohooks changes the value of the defect modulo 4 , so in type $D_{n}$ interchanges the twisted and untwisted types), and that $s$ is a fortiori regular in $\mathbf{L}$. Thus, the above result gives a recursive algorithm to compute the character values on all regular semisimple elements.

In the case of degenerate symbols we only get the values of the sum of two unipotent characters. These unipotent characters form one-element Lusztig families and so their values on regular semisimple classes are the values of the corresponding degenerate irreducible characters of the Weyl group; see [12, (4.6.10)]. The two characters for a degenerate symbol have the same values on non-degenerate classes, but different values on the degenerate classes. See [15] for a definition of unique parameters for the degenerate characters and classes and a formula to compute the missing values on degenerate classes. 
The following vanishing result is immediate from the above:

Corollary 3.4. Let $\mathbf{G}, \mathcal{S}$ and $\rho_{\mathcal{S}}$ be as in Theorem 3.3, and let $s \in G$ be a regular semisimple element lying in an F-stable maximal torus parameterised by $(\lambda, \mu)$. Then $\rho_{\mathcal{S}}(s)=0$ in any of the two following cases:

(1) $\lambda$ has a part of length $d$, but $\mathcal{S}$ does not have a d-hook; or

(2) $\mu$ has a part of length $d$, but $\mathcal{S}$ does not have a d-cohook.

Thus, if $\rho_{\mathcal{S}}(s) \neq 0$ and $(\lambda, \mu)=\left(\left(\lambda_{1}, \ldots, \lambda_{r}\right),\left(\mu_{1}, \ldots, \mu_{s}\right)\right)$, then it is possible to remove $\lambda_{i}$-hooks for $1 \leq i \leq r$ and $\mu_{i}$-cohooks for $1 \leq i \leq s$ from $\mathcal{S}$ in any chosen order.

We remark that we have computed many character values on semisimple classes for classical groups of rank $\leq 10$ by Deligne-Lusztig theory. These examples helped to find the statements given in the next section, and they also provided an independent check of the Murnaghan-Nakayama formula, Theorem 3.3 .

\section{VANISHING OF CHARACTERS}

In this section we prove Theorem 1 for quasi-simple groups of classical type. So let $\mathbf{G}$ be a simply-connected simple algebraic group of type $B_{n}$ with $n \geq 3, C_{n}$ with $n \geq 2$ or $D_{n}$ with $n \geq 4$, and let $F$ be a Steinberg morphism of $\mathbf{G}$ (we exclude the ${ }^{3} D_{4}$ case) with $G=\mathbf{G}(q):=\mathbf{G}^{F}$. We consider the corresponding finite groups $G=\operatorname{Spin}_{2 n+1}(q)$ only for odd $q$, and $\operatorname{Sp}_{2 n}(q)$ and $\operatorname{Spin}_{2 n}^{ \pm}(q)$ for any $q$, respectively.

We consider primes $\ell$ such that the Sylow $\ell$-subgroups of $G$ are not cyclic. We will show that for such $\ell$ with only very few exceptions all ordinary irreducible characters of $G$ vanish on some $\ell$-singular regular semisimple element.

If we write $d:=d_{\ell}(q)$ for the order of $q$ modulo $\ell$ as in Section 2.2 and then $n=a d+r, 0 \leq r<d$, for odd $d$, respectively, $n=a e+r, 0 \leq r<e$, for even $d=2 e$, then our assumption on $\ell$ implies $a \geq 2$ because the cyclotomic polynomial $\Phi_{d}(q)$ must divide the order of $\mathbf{G}(q)$ at least twice. If $G$ is of type ${ }^{2} D_{n}$ we have $n>2 d$ for odd $d$ and $n>2 e$ for even $d=2 e$ (see the order formula in [13, Tab. 24.1]).

4.1. Non-unipotent characters. We first deal with non-unipotent characters, and afterwards examine in more detail the unipotent characters.

Theorem 4.1. Let $G$ be one of the groups $\operatorname{Spin}_{2 n+1}(q)$ for odd $q$ and $n \geq 3, \operatorname{Sp}_{2 n}(q)$ for any $q$ and $n \geq 2$, or $\operatorname{Spin}_{2 n}^{ \pm}(q)$ for any $q$ and $n \geq 4$. Let $2 \neq \ell \backslash \chi q$ be a prime such that the Sylow $\ell$-subgroups of $G$ are non-cyclic. Then any non-unipotent irreducible complex character of $G$ vanishes on some $\ell$-singular regular semisimple element, except for the two cases $G=C_{2}(2)=\mathrm{Sp}_{4}(2)$ and $G=C_{4}(2)=\mathrm{Sp}_{8}(2)$.

The group $\mathrm{Sp}_{4}(2) \cong \mathfrak{S}_{6}$ contains no 3 -singular regular semisimple elements. It has three non-trivial irreducible characters which do not vanish on any 3-singular class, the unipotent character $\left(\begin{array}{c}012 \\ -\end{array}\right)$ and the two characters of degree 10 (belonging to Lusztig series of type $\left.A_{1}(q) \times(q+1)\right)$.

The group $\mathrm{Sp}_{8}(2)$ has only one 5-singular regular semisimple class and there are 15 irreducible characters which do not vanish on this class. There is only one non-trivial character which does not vanish on any 5-singular class, the unipotent character $\left(\begin{array}{c}01 \\ 4\end{array}\right)$.

Proof. We use the following facts from Deligne-Lusztig theory. The irreducible characters of $G$ are partitioned into Lusztig series which are parameterised by 
semisimple conjugacy classes of the dual group $G^{*}$. If $s \in G^{*}$ is semisimple then the values of characters in the Lusztig series of $s$ on semisimple elements $t \in G$ are linear combinations of values of Deligne-Lusztig characters on $t$ which belong to types of tori which occur in the connected centraliser of $s$ in $\mathbf{G}^{*}$. In particular Deligne-Lusztig characters corresponding to a maximal torus $T$ of $G$ vanish on regular semisimple classes which do not intersect $T$; see e.g. [11, Prop. 6.4].

Since $\mathbf{G}$ is simply-connected the dual group $\mathbf{G}^{*}$ has trivial center so that nonunipotent characters are in Lusztig series of non-central elements.

We show the theorem by listing in each case a set of types of maximal tori which contain regular $\ell$-singular elements by Lemma 2.1 and which cannot all occur in any proper centraliser. We have described these subgroups together with the types of tori they contain in Lemma 2.3. Note that for $\mathbf{G}$ of type $B_{n}$ the dual group is of type $C_{n}$ (we need to apply Lemma 2.3 for type $C_{n}$ and consider tori with regular elements in type $B_{n}$ ); the same remark holds with $B_{n}$ and $C_{n}$ interchanged.

Let $d=d_{\ell}(q)$ and write $n=a d+r$ with $0 \leq r<d$ for odd $d$ and $n=a e+r$ with $0 \leq r<e$ for even $d=2 e, a \geq 2$, as explained above.

Recall that for odd $d$ a torus of type $(\lambda, \mu)$ contains $\ell$-singular elements if and only if some part of $\lambda$ is a positive multiple of $d$. For even $d=2 e$ a torus of type $(\lambda, \mu)$ contains $\ell$-singular elements if and only if $\mu$ contains an odd multiple of $e$ or $\lambda$ contains an even multiple of $e$.

We collect the tori we consider in Table 1 .

A few additional remarks are in order. In the two cases marked with $\left(^{*}\right)$ in the table we need to rule out the possibility that the given tori all lie in a subgroup of type $D_{d}+D_{n-d}$ or $D_{e}+D_{n-e}$, respectively. Such a subgroup stabilises a decomposition of the orthogonal space on which $G$ acts into two non-degenerate subspaces. In the first case one of these subspaces must be of minus- and the other of plus-type. So the corresponding finite subgroups are of type ${ }^{2} D_{d}+D_{2 d}$ or $D_{d}+{ }^{2} D_{2 d}$, each of these only contains one of the types of tori, $((d),(n-d))$ or $((n-d),(d))$. The argument for the second case is similar; there, both subspaces must be of minus-type or both of plus-type.

It remains to check for which groups one of the tori given in Table 1 does not contain regular elements. Note that for $q=3$ we have $d>2$ because $\ell \neq 2$, and for $q=2$ we have $d>1$. Furthermore, when $r>0$, we have $d \geq 3$ or $e \geq 2$, respectively. This implies that only the following cases need an extra consideration:

$C_{2}(2)$ with $e=1$ : this is the first exception mentioned in the statement.

$B_{4}(q)$ with $q=3$ and $e=2$ : We compute that for $q=3$ the torus of type $((1),(1,2))$ contains a regular element. So, we can argue with tori of types $((4),-)$ and $((1),(1,2))$.

$C_{4}(q)$ with $q=3$ and $e=2$ : In this case the torus of type $((2),(2))$ in $C_{4}(3)$ contains regular elements. Here we can argue with tori of types $((4),-)$ and $((2),(2))$ by checking that the dual group (adjoint of type $\left.B_{4}(3)\right)$ does not contain a semisimple element whose centraliser contains tori of both types.

For $C_{4}(2)$ and $e=2$ (and so $\ell=5$ ) only tori of type $((4),-)$ contain $\ell$-singular regular elements. This leads to the second exception mentioned in the statement.

${ }^{2} D_{4}(2)$ with $e=1$ : Here, the maximal tori containing $\ell$-elements all have conjugates inside a subgroup of type $D_{1}+D_{3}$. We can apply the argument from above: the tori of types $((1),(3))$ and $((3),(1))$ cannot both occur in the same rational form of such a subgroup. 
TABLE 1. Tori needed in the proof of Theorem 4.1

\begin{tabular}{|c|c|c|c|c|}
\hline type & $d$ & $a$ & $r$ & tori \\
\hline$C_{n}, B_{n}$ & odd & any & 0 & $(1, d-1))$ if $d>1$ \\
\hline$C_{n}, B_{n}$ & odd & any & $>0$ & $\begin{array}{l}((n-r),(r)),((n-r-d),(d+r)),((n-r-d), \\
(d+r-1,1))\end{array}$ \\
\hline$D_{n}$ & odd & any & 0 & $\begin{array}{l}((n),-),((n-1,1),-) \text { if } d=1, \text { or }((n-d),(d-1,1)) \\
\text { if } d>1\end{array}$ \\
\hline$D_{n}$ & odd & any & $>0$ & $\begin{array}{l}((n-d-r, d+r),-),((n-r, r),-) \text { if } r \neq 2, \text { or } \\
((n-2),(1,1)) \text { if } r=2\end{array}$ \\
\hline${ }^{2} D_{n}\left({ }^{*}\right)$ & odd & any & 0 & $\begin{array}{l}((n-d),(d)),((d),(n-d)),((n-d-1, d),(1)) \text { if } \\
d>1\end{array}$ \\
\hline${ }^{2} D_{n}$ & odd & any & $>0$ & $\begin{array}{l}((n-r),(r)),((n-d-r),(d+r)),((n-d-r, 1) \\
(d+r-1))\end{array}$ \\
\hline$C_{n}, B_{n}$ & $=2 e$ & even & 0 & $((n),-),((n-e),(e)),(-,(n-e-1, e, 1))$ if $e>1$ \\
\hline$C_{n}, B_{n}$ & $=2 e$ & even & $>0$ & $\begin{array}{l}((n-r),(r)),(-,(n-e, e)),(-,(n-e-1, e, 1)) \text { if } \\
r \neq 1\end{array}$ \\
\hline$C_{n}, B_{n}$ & $=2 e$ & odd & 0 & $(-,(n)),(-,(n-e, e)),((n-e-1),(e, 1))$ if $e>1$ \\
\hline$C_{n}, B_{n}$ & $=2 e$ & odd & $>0$ & $\begin{array}{l}(-,(n-r, r)),((n-r-e),(r+e)),((n-r-e), \\
(r+e-1,1))\end{array}$ \\
\hline$D_{n}$ & $=2 e$ & even & 0 & $\begin{array}{l}((n),-),((n-e-1),(e, 1)) \text { if }(n, e) \notin\{(4,1),(6,3)\} \\
\text { or }((n-e-2),(e, 2)) \text { otherwise }\end{array}$ \\
\hline$D_{n}$ & $=2 e$ & even & $>0$ & $\begin{array}{l}(-,(n-e, e)),((n-1,1),-) \text { if } r=1,((n-r), \\
(r-1,1)) \text { if } r>1\end{array}$ \\
\hline$D_{n}\left({ }^{*}\right)$ & $=2 e$ & odd & 0 & $\begin{array}{l}(-,(n-e, e)),(-,(n-2 e, 2 e)),((1),(n-2 e, 2 e-1)) \\
\text { if } e>1,((2 e),(e-1,1) \text { if } n=3 e\end{array}$ \\
\hline$D_{n}$ & $=2 e$ & odd & $>0$ & $\begin{array}{l}(-,(n-r, r)),(-,(n-e, e)),((1),(n-r, r-1)) \text { if } \\
r>1\end{array}$ \\
\hline${ }^{2} D_{n}$ & $=2 e$ & even & 0 & $\begin{array}{l}((n-e),(e)),((n-2 e),(2 e)),((n-2 e, 2 e-1),(1)) \\
\text { if } e>1\end{array}$ \\
\hline${ }^{2} D_{n}$ & $=2 e$ & even & $>0$ & $((n-r),(r)),((n-e),(e)),((n-r, 1),(r-1))$ if $r>1$ \\
\hline${ }^{2} D_{n}$ & $=2 e$ & odd & 0 & $(-,(n)),((n-e),(e)),((n-e, 1),(e-1))$ if $e>1$ \\
\hline${ }^{2} D_{n}$ & $=2 e$ & odd & $>0$ & $\begin{array}{l}((n-e-r),(e+r)),(-,(n-r, r-1,1)) \text { if } r>1, \\
((1),(n-1)) \text { if } r=1\end{array}$ \\
\hline
\end{tabular}

4.2. Unipotent characters. In the following theorem we use symbols to describe unipotent characters and refer to Section 3.2 for the notation.

Theorem 4.2. Let $G$ be one of the groups $\operatorname{Spin}_{2 n+1}(q)$ for odd $q$ and $n \geq 3, \operatorname{Sp}_{2 n}(q)$ for any $q$ and $n \geq 2$, or $\operatorname{Spin}_{2 n}^{ \pm}(q)$ for any $q$ and $n \geq 4$. Let $2 \neq \ell \not \chi q$ be a prime such that the Sylow $\ell$-subgroups of $G$ are non-cyclic.

Let $\rho_{\mathcal{S}}$ be a unipotent character of $G$ for some symbol $\mathcal{S}$ and assume that $\rho_{\mathcal{S}}$ does not vanish on any regular semisimple $\ell$-singular class. Then either $\rho_{\mathcal{S}}$ is the trivial character, or the Steinberg character, or up to Alvis-Curtis duality we are in one of the following cases (as before we set $d=d_{\ell}(q)$, and interpret -1 as the empty set): 
(1) $G=\operatorname{Sp}_{2 n}(q)$ or $\operatorname{Spin}_{2 n+1}(q)$, d is odd, $n=2 d+r$ with $0 \leq r<d$ and

$$
\mathcal{S}=\left(\begin{array}{c}
\frac{d-r-1}{\underline{d-r}-1} 0 \cup d \cup 2 d \\
\underline{2 e}
\end{array}\right) ;
$$

(2) $G=\operatorname{Sp}_{2 n}(q)$ or $\operatorname{Spin}_{2 n+1}(q), d=2 e$ is even, $n=2 e+r$ with $0 \leq r<e$ and

$$
\mathcal{S}=\left(\begin{array}{c}
\frac{e-r-1}{e-r-1 \backslash} \backslash 0 \cup 2 e \\
\underline{e-r}
\end{array}\right) ;
$$

(3) $G=\operatorname{Spin}_{2 n}^{+}(q), d$ is odd, $n=2 d+r$ with $0 \leq r<d$ and

$$
\mathcal{S}=\left(\begin{array}{c}
\frac{d-r-1}{\underline{d}-r} \mathbf{x} \cup d \cup 2 d
\end{array}\right) ;
$$

(4) $G=\operatorname{Spin}_{2 n}^{+}(q), d=2 e$ is even, $n=2 e+r$ with $0 \leq r \leq e$ and

$$
\mathcal{S}=\left(\begin{array}{c}
\frac{e-r-1}{e-r} \backslash 0 \cup 2 e \\
\underline{e-r}
\end{array}\right) ;
$$

(5) $G=\operatorname{Spin}_{2 n}^{-}(q)$, $d$ is odd, $n=2 d+r$ with $0<r \leq d$ and

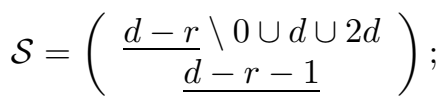

(6) $G=\operatorname{Spin}_{2 n}^{-}(q), d=2 e$ is even, $n=2 e+r$ with $0<r<e$ and

$$
\mathcal{S}=\left(\begin{array}{c}
\underline{e-r} \cup e \\
\underline{e-1} \backslash 0 \cup 2 e
\end{array}\right)
$$

(7) $G=\operatorname{Sp}_{4}(2), d=2$ and $\mathcal{S}=\left(\begin{array}{ccc}0 & 1 & 2 \\ - & -\end{array}\right)$ or $\left(\begin{array}{cc}0 & 2 \\ 1\end{array}\right)$ (there is no regular semisimple $\ell$-singular element);

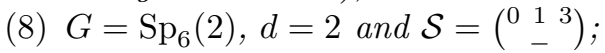

(9) $G=\mathrm{Sp}_{8}(2), d=4$ and $\mathcal{S}=\left(\begin{array}{cc}0 & 1 \\ 4\end{array}\right)$ or $\left(\begin{array}{cc}1 & 4 \\ 0\end{array}\right)$; or

(10) $G=\operatorname{Spin}_{8}^{-}(2), d=2,4$ and $\mathcal{S}=\left(\begin{array}{cc}1 & 3 \\ -\end{array}\right)$.

Conversely, all characters listed above take non-zero values on all regular semisimple $\ell$-singular classes. More precisely, in cases $G=\operatorname{Spin}_{8}^{-}(2), d=2,4, \mathcal{S}=\left(\begin{array}{cc}1 & 3 \\ -\end{array}\right)$ the character $\rho_{\mathcal{S}}$ has value 2 on classes of type $(-,(2,1,1))$. In all other cases the character values are \pm 1 .

Proof. (a) Our strategy is to use Corollary 3.4 of the Murnaghan-Nakayama formula. We consider maximal tori with $\ell$-singular regular elements which are parameterised by pairs of partitions with very few parts. For example, if for a symbol $\mathcal{S}$ the character $\rho_{\mathcal{S}}$ has non-zero value on regular elements in a torus of type $\left(\left(\lambda_{1}\right),\left(\mu_{1}\right)\right)$ then the Murnaghan-Nakayama formula implies that we can remove a $\lambda_{1}$-hook from $\mathcal{S}$ and that the resulting symbol has a $\mu_{1}$-cohook which after removing leaves a symbol of rank 0 .

(b) For groups of type $B_{n}$ and $C_{n}$ we need to consider symbols of rank $n$ with odd defect. First note that there is only one symbol of rank 0 with odd defect, namely the defect 1 symbol

$$
\left(\begin{array}{c}
0 \\
-
\end{array}\right)=\left(\begin{array}{c}
\underline{n} \\
\underline{n-1}
\end{array}\right)
$$

(apply the shift operation $n$ times). We will write all symbols with $2 n+1$ entries, because this way we can construct all symbols of rank $n$ by adding hooks or cohooks starting from the rank 0 symbol without the need of further shift operations. Also, 
we will write all symbols of non-zero defect such that the larger number of entries is in the first row.

The symbols of rank $t \leq n$ which have a $t$-hook are $\left(\begin{array}{c}\underline{n} \backslash k \cup k+t \\ n-1\end{array}\right)$ for $n+1-t \leq k \leq n$ and their dual symbols $\left({ }_{n-1} \backslash \frac{n}{k} \cup k+t\right)$ for $n-t \leq k \leq n-\overline{1}$.

To find up to duality all symbols $\mathcal{S}$ of rank $n$ which have an $(n-t)$-cohook $c$ such that $\mathcal{S} \backslash c$ has a $t$-hook, it is sufficient to take the first set of symbols of rank $t$ with a $t$-hook and to add an $(n-t)$-cohook in all possible ways (filling the gap $k$, creating new entries in the first or the second row or moving the $k+t$ entry). We get:

$$
\begin{array}{cl}
\left(\begin{array}{c}
\underline{n} \cup l+n \\
\underline{n}-1 \backslash l
\end{array}\right) & (1 \leq l \leq t), \\
\left(\begin{array}{c}
\underline{n} \backslash k \cup k+t \cup l+n-t \\
\underline{n-1} \backslash l
\end{array}\right) & (n-t<k \leq n, t<l<n, k-l \neq n-2 t), \\
\left(\begin{array}{c}
\frac{n-1}{n} \cup l+n-t \\
\underline{n} \backslash k \cup k+t
\end{array}\right) & (n-t<k \leq n, t \leq l \leq n, k \neq l), \\
\left(\begin{array}{c}
\frac{n-1}{\underline{n}} \backslash k+n \\
\underline{n} \backslash k
\end{array}\right) & (n-t<k \leq n) .
\end{array}
$$

(c) We now prove the theorem for the case $B_{n}, C_{n}$ for $n \geq 3$ and odd $d$. Write $n=a d+r$ with $0 \leq r<d$. Note that for $q=2$ or 3 we have $d>1$ (since $\ell \neq 2$ ). In our argument we will use maximal tori which by Lemma 2.1 contain regular $\ell$-singular elements.

First assume $r=0$ and let $\mathcal{S}$ be a symbol of rank $n$ such that $\rho_{\mathcal{S}}$ is non-zero on all regular semisimple $\ell$-singular classes. Then as a torus of type $((n),-)$ contains $\ell$-singular elements, $\mathcal{S}$ has an $n$-hook, that is (up to duality) one of

$$
\mathcal{S}=\left(\begin{array}{c}
\underline{n} \backslash k \cup k+n \\
\underline{n-1}
\end{array}\right) \quad(1 \leq k \leq n) .
$$

Tori of type $((d),(n-d))$ also contain $\ell$-singular regular elements. Therefore $\mathcal{S}$ must have a $d$-hook $h$ and an $(n-d)$-cohook such that $\mathcal{S} \backslash h$ still has an $(n-d)$-cohook. This is only the case for $k=n$ and $k=n-d$. For $k=n$ we obtain the symbol $\left(\frac{n-1 \cup 2 n}{\underline{n}-1}\right)$ of the trivial character. For $k=n-d$ we get the symbol

$$
\mathcal{S}=\left(\begin{array}{c}
\underline{n} \backslash n-d \cup 2 n-d \\
\underline{n-1}
\end{array}\right) .
$$

If $a>2$ then this symbol has no $(a-1) d=(n-d)$-hook, hence $\rho_{\mathcal{S}}$ is zero on regular elements in tori of type $((n-d),(d))$. For $a=2$ we get one of the symbols shown in part (1) of the statement (after applying the inverse shift operation $n-d$ times).

Now let $r>0$ (and so $d \geq 3)$. We consider tori of types $((a d),(r))=((n-r),(r))$ and $((d, n-d),-)$. Here we check for every symbol $\mathcal{S}$ listed in (b) (for $t=n-r$ ) if it has an $(n-r)$-hook and if we can get the rank 0 symbol by removing a $d$ hook and an $(n-d)$-hook in both possible orders. Note that the last condition implies that $\mathcal{S}$ has defect one because removing hooks does not change the defect. These conditions yield in all cases relations between the parameters $k$ and $l$ and then determine $k$. The only possible symbols which remain are those of the trivial 
character and

$$
\mathcal{S}=\left(\begin{array}{c}
\underline{n} \\
\underline{n} \backslash d \backslash n-r) n+d-r
\end{array}\right) .
$$

If $a>2$ this has no $(a-1) d$-hook and so $\rho_{\mathcal{S}}$ is zero on regular elements in tori of type $(((a-1) d),(d+r))$. For $a=2$ the dual of $\mathcal{S}$ is given in statement (1), that is,

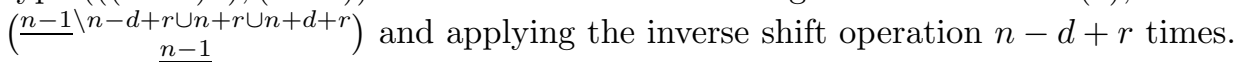

(d) The argument for type $B_{n}, C_{n}, n \geq 7$, and even $d=2 e, n=a e+r$, is very similar. Pairs of partitions corresponding to tori with $\ell$-singular regular elements must now have an odd multiple of $e$ in the second part or an even multiple of $e$ in the first part. Therefore we distinguish the cases of even and odd $a$ and of $r=0$ and $r>0$. We just list the types of tori which we consider in the given order such that the arguments from (c) work with slight modifications.

$a$ even, $r=0$ : $((n),-)$ and $((n-e),(e))$, and for $a>2$ also $((n-2 e),(2 e))$.

$a$ even, $r>0$ : $((n-r),(r)),(-,(n-e, e))$ and $((n-e),(e))$, and for $a>2$ also $(-,((a-1) e, e+r))$.

$a$ odd, $r=0$ : $((n-e),(e)),(-,(n))$ and $(-,(n-e, e))$, and finally to rule out a symbol of defect 3 also $((n-e, e),-)$ (if $a \leq 3)$ or $((n-3 e),(3 e))$ (if $a>3)$.

$a$ odd, $r>0$ : $((n-r-e),(e+r)),(-,(n-r, r))$ and $((n-e),(e))$.

(e) The cases of type $D_{n}$ and ${ }^{2} D_{n}$ can also be discussed with very similar arguments. The only symbol of rank 0 and even defect has defect 0 and is $\left(\begin{array}{l}- \\ -\end{array}\right)=\left(\frac{n-1}{n-1}\right)$ and so all the symbols of rank $n$ with an $n$-hook are

$$
\mathcal{S}=\left(\begin{array}{l}
\underline{n-1} \backslash k \cup k+n \\
\underline{n-1}
\end{array}\right) \quad(0 \leq k \leq n)
$$

Here it is sufficient to consider the cases with $k \geq(n-1) / 2$ in further arguments because the remaining ones are the duals of these.

(f) Type $D_{n}, n \geq 7$. We list again which tori we consider in the various cases.

$d$ odd, $r=0$ : $((n),-)$ and in case $d=1$ we use $((n-1,1),-)$ and for $d \geq 3$ we use $((n-d),(d-1,1))$. For $a>2$ we also need $((n-2 d),(2 d-1,1))$ to rule out one possibility.

$d$ odd, $r>0:((n-d, d),-)$ and $((n-r, r),-)$ if $r \neq 2$ or $((n-2),(1,1))$ if $r=2$, $((d),(n-d-1,1))$.

$d=2 e$ even, $n=a e, a$ even: $((n),-)$ and $(-,(n-1,1))$ if $e=1$ or $((n-e-$ $1),(e, 1))$ if $e>1$. For $a>2$ we also use $((n-2 e),(2 e-1,1))$.

$d=2 e$ even, $n=a e+r, a$ even, $r>0:(-,(n-e, e)),((n-e-1),(e, 1))$ and $((n-r, r),-)$ if $r \neq 2$ or $((n-2),(1,1))$ if $r=2$. For $a>2$ we also need $((2 e),(n-2 e-1,1))$.

$d=2 e$ even, $n=a e, a$ odd: $(-,(n-e, e))$ and $((n-e, e),-)$ if $e \neq 2$ or $((n-2),(1,1))$ if $e=2$. Finally, use $((n-3 e, 3 e),-)$ if $a>3$. For $a=3$ we get the exception listed in (4) for the parameter $r=e$ (that is, $n=2 e+r=3 e$ ).

$d=2 e$ even, $n=a e+r, a$ odd, $r>0:(-,(n-e, e)),(-,(n-r, r)),((n-e-$ $1),(e, 1))$ and $((2 e),(n-2 e-1,1))$.

(g) Type ${ }^{2} D_{n}, n \geq 7$. We list again which tori we consider in the various cases.

$d$ odd, $n=a d$ : Recall that in this case we have $a \geq 3$. We use $((n-d),(d))$ and $((n-2 d),(2 d))$. For $a>3$ we also use $((n-3 d),(d))$ and for $a=3$ we get the exception listed in (5) for $r=d$ (that is $n=2 d+r=3 r$ ). 
$d$ odd, $n=a d+r, r>0$ (and so $d \geq 3):((n-r),(r)),((d),(n-d))$ and $((d, 1),(n-d-1))$, and for $a>2$ also $((2 d),(n-2 d))$. For $a=2$ this leads to the other exceptions listed in (5).

$d=2 e$ even, $n=a e, a$ even: Recall that in this case we have $a \geq 4$. We use $((n-e),(e)),((n-2 e),(2 e))$ and one of $((3),(n-3))$ (if $e=1)$ or $((n-3 e),(3 e))$ (if $e>1)$ or $(-,(6,1,1)$ ) (if $n=8)$.

$d=2 e$ even, $n=a e+r, a$ even, $r>0$ (so $e>1)$ : $((n-e),(e))$ and $((n-r),(r))$. For $a>2$ we also use $((2 e),(n-2 e))$. For $a=2$ this leads to the exceptions listed in $(6)$.

$d=2 e$ even, $n=a e, a$ odd: $(-,(n)),((n-e),(e))$ and $((n-e-1,1),(e))$.

$d=2 e$ even, $n=a e+r, a$ odd, $r>0:((n-e),(e)),((n-e-r),(e+r))$ and $((2 e),(n-2 e))$. Finally, also $((r),(n-r))$ if $r \neq 2$ or $(-,(n-2,1,1))$ for $r=2$.

(h) For small rank $n \leq 6$ we compute the number of regular elements in all tori, and determine the values of unipotent characters on all $\ell$-singular regular semisimple classes via the Murnaghan-Nakayama formula in Theorem 3.3 . This yields the extra cases in statements (7) to (10).

(i) The symbols given in statements (1) to (6) each contain a unique $d$-hook and a unique $2 d$-hook (or a unique $e$-cohook and unique $2 e$-hook, respectively). Removing these we always get the symbol of the trivial representation of the appropriate rank (and the Steinberg representation for the dual symbols). Therefore, the Murnaghan-Nakayama formula shows that the values on all regular semisimple $\ell$-singular elements are \pm 1 .

\section{Simple Endotrivial modules}

In this section we prove Theorem 2 on the classification of simple endotrivial modules for finite classical groups. Note that the case of linear and unitary groups has already been treated in [10], so we only need to consider symplectic groups and spin groups as in the previous section.

5.1. On certain unipotent characters. We first rule out some candidate characters. Let $\Phi_{d} \in \mathbb{Z}[x]$ denote the $d$ th cyclotomic polynomial.

Proposition 5.1. Let $\chi$ be the unipotent character of $G=\mathrm{SO}_{6 d-1}(q)$ or $\operatorname{Sp}_{6 d-2}(q)$ (respectively of $\left.\mathrm{SO}_{2(3 d-1)}^{+}(q), \mathrm{SO}_{6 d}^{-}(q)\right)$ parameterised by the symbol

$$
\left(\begin{array}{c}
d \\
2 d \\
0
\end{array}\right) \quad \text { respectively }\left(\begin{array}{cc}
d & 2 d \\
0 & 1
\end{array}\right),\left(\begin{array}{ll}
d & 2 d \\
\end{array}\right)
$$

with $d \geq 3$ odd, or the unipotent character of $\mathrm{SO}_{6 e}^{+}(q)$ parameterised by

$$
\left(\begin{array}{c}
2 e \\
e
\end{array}\right)
$$

for $d=2 e$ even. Then $\chi(1) \not \equiv \pm 1\left(\bmod |G|_{\ell}\right)$ for any prime $2<\ell$ with $d_{\ell}(q)=d$.

Proof. Since $\Phi_{d}(q)$ divides $|G|$ at least twice it is sufficient to show that $\chi(1) \not \equiv \pm 1$ $\left(\bmod \Phi_{d}(q)_{\ell}^{2}\right)$. We use a version of Babbage's congruence for quantum binomial coefficients

$$
\left[\begin{array}{c}
h d-1 \\
d-1
\end{array}\right]_{x} \equiv x^{(h-1)\left(\begin{array}{l}
d \\
2
\end{array}\right)} \quad\left(\bmod \Phi_{d}(x)^{2}\right) \quad \text { in } \mathbb{Z}[x]
$$


for any $d, h \geq 2$ (proved in [10, Lemma 3.7]). If $\chi$ belongs to $\left(\begin{array}{c}d \\ 2 d \\ 0\end{array}\right)$, we can express its degree using a quantum binomial coefficient evaluated at $q^{2}$ (see [4, 13.8]):

$$
\chi(1)=\frac{\left(q^{2}-1\right) \cdots\left(q^{6 d-2}-1\right)\left(q^{2 d}-q^{d}\right)\left(q^{2 d}+1\right)\left(q^{d}+1\right)}{2\left(q^{2}-1\right) \cdots\left(q^{4 d}-1\right)\left(q^{2}-1\right) \cdots\left(q^{2 d}-1\right)}=\frac{1}{2}\left[\begin{array}{c}
3 d-1 \\
d-1
\end{array}\right]_{q^{2}} q^{d}\left(q^{2 d}+1\right) .
$$

Substituting $x=q^{2}$ in the Babbage congruence we get a congruence over the integers modulo $\Phi_{d}\left(q^{2}\right)^{2}$. Since $\Phi_{d}\left(x^{2}\right)=\Phi_{d}(x) \Phi_{d}(-x)$ we also get a congruence modulo $\Phi_{d}(q)^{2}$ :

$$
\chi(1) \equiv \frac{1}{2} q^{2 d(d-1)+d}\left(q^{2 d}+1\right) \quad\left(\bmod \Phi_{d}(q)^{2}\right) .
$$

It suffices to show that $2 \chi(1) \not \equiv \pm 2\left(\bmod \Phi_{d}(q)_{\ell}^{2}\right)$. Since $\Phi_{d}(q) \mid\left(q^{d}-1\right)$ we get

$$
\begin{aligned}
2 \chi(1) & \equiv q^{2 d(d-1)+d} \cdot 2 q^{d}=2\left(q^{2 d}\right)^{d} \equiv 2\left(q^{2 d}-\left(q^{d}-1\right)^{2}\right)^{d}=2\left(2\left(q^{d}-1\right)+1\right)^{d} \\
& \equiv 2\left(2 d\left(q^{d}-1\right)+1\right) \quad\left(\bmod \Phi_{d}(q)^{2}\right) .
\end{aligned}
$$

Since $\ell \neq 2, d<\ell$ and $\ell \mid\left(q^{d}-1\right)$ we conclude that $2 \chi(1)+2$ is not divisible by $\ell$ and that the highest power of $\ell$ dividing $2 \chi(1)-2$ is $\left(q^{d}-1\right)_{\ell}=\Phi_{d}(q)_{\ell}$.

Now we turn to the unipotent character with symbol $\left(\begin{array}{cc}d & 2 d \\ 0 & 1\end{array}\right)$. Its degree differs from the previously considered $\chi(1)$ by the factor

$$
\frac{\left(q^{2 d}+q\right)\left(q^{d-1}+1\right)}{\left(q^{3 d-1}+1\right)(q+1)}=\frac{q^{3 d-1}+3 q^{d}+q-1+\left(q^{d}-1\right)^{2}}{q^{3 d-1}+3 q^{d}+q-1+\left(q^{d}+2\right)\left(q^{d}-1\right)^{2}} .
$$

We argue that both degrees are equal modulo $\Phi_{d}(q)_{\ell}^{2}$.

For this, note that for integers $a, b, c, m$ with $a b / c \in \mathbb{Z}, b \equiv c(\bmod m)$ and $(c, m)=1$ there is an integer $\tilde{c}$ with $c \tilde{c} \equiv b \tilde{c} \equiv 1(\bmod m)$ and so $a b / c \equiv a b / c \cdot c \tilde{c} \equiv$ $a b \tilde{c} \equiv a(\bmod m)$. This applies here because $\left(q^{3 d-1}+1\right)(q+1)$ is not divisible by $\ell$ (since $d \geq 3$ is the order of $q$ modulo $\ell$ and $\left(q^{3 d-1}+1\right)(q+1)\left(q^{3 d-1}-1\right)(q-1)=$ $\left(q^{6 d-2}-1\right)\left(q^{2}-1\right)$ is not divisible by $\left.\ell\right)$.

The same argument works for the degree of the unipotent character with symbol $\left(\begin{array}{ll}d & 2 d\end{array}\right)$. Its degree differs from the previous $\chi(1)$ by the factor

$$
\frac{2\left(q^{2 d}-q^{d}+1\right)}{q^{2 d}+1}=\frac{2 q^{d}+2\left(q^{d}-1\right)^{2}}{2 q^{d}+\left(q^{d}-1\right)^{2}}
$$

and $q^{2 d}+1$ is not divisible by $\ell$.

Finally, we consider for even $d=2 e$ the unipotent character $\psi$ with symbol $\left(\begin{array}{c}2 e \\ e\end{array}\right)$. Its degree is

$$
\psi(1)=\left[\begin{array}{c}
3 e-1 \\
e-1
\end{array}\right]_{q^{2}}\left(q^{2 e}+q^{e}+1\right) q^{e} .
$$

Evaluating Babbage's congruence at $q^{2}$ yields a congruence modulo $\Phi_{e}\left(q^{2}\right)^{2}$. But note that for odd $e$ we have $\Phi_{e}\left(x^{2}\right)=\Phi_{e}(x) \Phi_{e}(-x)$ and $\Phi_{e}(-x)=\Phi_{d}(x)$, and for even $e$ we have $\Phi_{e}\left(x^{2}\right)=\Phi_{d}(x)$. So, we also get congruences modulo $\Phi_{d}(q)^{2}$. Using now $\Phi_{d}(q) \mid\left(q^{e}+1\right)$ we find with similar arguments as before that $\psi(1) \equiv-q^{2 e^{2}}$ $\left(\bmod \Phi_{d}(q)^{2}\right)$ and that $\psi(1)-1$ is not divisible by $\ell$, and that $\psi(1)+1$ is divisible by $\Phi_{d}(q)_{\ell}$ but no higher power of $\ell$.

In order to deal with certain unipotent characters, we recall some results of James and Mathas [8] on reducibility of characters of Hecke algebras. Let $\mathcal{H}_{n}=\mathcal{H}_{n}(q, Q)$ be the Iwahori-Hecke algebra of type $B_{n}$ with parameters $q$ and $Q$ over $\mathbb{Z}\left[q^{ \pm 1}, Q^{ \pm 1}\right]$. Its irreducible characters are in natural bijection with those of the Weyl group 
$W\left(B_{n}\right)$ via the specialisation $q \mapsto 1, Q \mapsto 1$, and hence labelled by pairs of partitions $(\lambda, \mu)$ of $n$. We write $\chi_{\lambda, \mu}$ for the character of $\mathcal{H}_{n}$ labelled by $(\lambda, \mu)$. We consider certain specialisations of $\mathcal{H}_{n}$.

Proposition 5.2. Let $\ell$ be a prime and $q$ a prime power. Set $d=d_{\ell}(q)$.

(a) If $d$ is odd, $n=2 d+r$ with $0 \leq r \leq d-2$, and $(\lambda, \mu)=((d+r, r+$ $\left.\left.1,1^{d-r-1}\right),-\right)$, then the character $\chi_{\lambda, \mu}$ of $\mathcal{H}_{n}(q, q)$ is reducible modulo $\ell$.

(b) If $d=2 e>2$ is even, $n=2 e+r$ with $0 \leq r \leq e-1$, and $(\lambda, \mu)=$ $\left((r),\left(e+r+1,1^{e-r-1}\right)\right)$, then the character $\chi_{\lambda, \mu}$ of $\mathcal{H}_{n}(q, q)$ is reducible modulo $\ell$.

Proof. Let $\zeta_{d}$ denote a primitive complex $d$ th root of unity. The reduction modulo $\ell$ of $\mathcal{H}_{n}(q, q)$ factors through the specialisation to $\mathcal{H}_{n}\left(\zeta_{d}, \zeta_{d}\right)$, thus a character which becomes reducible under the latter specialisation will a fortiori be reducible modulo $\ell$. James and Mathas [8, Thms. 4.7(i) and 4.10] give a sufficient criterion for $\chi_{\lambda, \mu}$ to become reducible under the specialisation to $\mathcal{H}_{n}\left(\zeta_{d}, \zeta_{d}\right)$, if $(\lambda, \mu)$ is a Kleshchev bipartition. In case (a), $\chi_{\lambda, \mu}$ is reducible modulo $\Phi_{d}$ by [8, Thm. 4.10(i)] since the character of the Hecke algebra of $\mathfrak{S}_{n}$ labelled by $\lambda=\left(d+r, r+1,1^{d-r-1}\right)$ is reducible modulo $\Phi_{d}$, as the first column hook lengths are different.

In (b), $(\lambda, \mu)$ is Kleshchev, and for $r>0$ the last box of the Young diagram of $\lambda$ can be moved to the end of the first column of $\mu$ without changing its residue, so $\chi_{\lambda, \mu}$ is reducible by [8, Thm. 4.10(ii)]. For $r=0$ the character $\chi_{\lambda, \mu}$ is reducible modulo $\Phi_{2 e}$ by [8, Thm. 4.10(i)] since the character of the Hecke algebra of $\mathfrak{S}_{n}$ labelled by the second part $\mu=\left(e+1,1^{e-1}\right)$ is.

The next reducibility result for characters of specialisations of the Hecke algebra of type $B_{n}$ will be used in the investigation of unipotent characters of $\operatorname{Spin}_{2 n}^{ \pm}(q)$.

Proposition 5.3. Let $\ell$ be a prime and $q$ a prime power. Set $d=d_{\ell}(q)$.

(a) If $d$ is odd, $n=2 d+r$ with $0 \leq r \leq d-2$, and $(\lambda, \mu)=((d+r, r+$ $\left.\left.1,1^{d-r-1}\right),-\right)$, then the character $\chi_{\lambda, \mu}$, of $\mathcal{H}_{n}(q, 1)$ is reducible modulo $\ell$.

(b) If $d=2 e>2$ is even, $n=2 e+r$ with $0 \leq r \leq e-1$, and $(\lambda, \mu)=$ $\left(\left(e+r, 1^{e-r}\right),(r)\right)$, then the character $\chi_{\lambda, \mu}$ of $\mathcal{H}_{n}(q, 1)$ is reducible modulo $\ell$.

(c) If $d$ is odd, $n=2 d+r$ with $1 \leq r \leq d-1$, and $(\lambda, \mu)=\left(d+r-1, r, 1^{d-r}\right),-$, then the character $\chi_{\lambda, \mu}$ of $\mathcal{H}_{n-1}\left(q, q^{2}\right)$ is reducible modulo $\ell$.

(d) If $d=2 e>2$ is even, $n=2 e+r$ with $1 \leq r \leq e-1$, and $(\lambda, \mu)=$ $\left((r-1),\left(e+r+1,1^{e-r-1}\right)\right)$, then the character $\chi_{\lambda, \mu}$ of $\mathcal{H}_{n-1}\left(q, q^{2}\right)$ is reducible modulo $\ell$ unless $(e, r)=(2,1)$.

Proof. Parts (a) and (c) again follow from [8, Thm. 4.10(i)] as the first column hook lengths of $\lambda$ are different. The characters $\chi_{\lambda, \mu}$ as in (b) and (d) are reducible since the first, respectively, second part of $\lambda$ is a hook partition, of length $d=2 e$, hence belonging to a reducible character of the Hecke algebra of type $\mathfrak{S}_{2 e}$, unless this hook is either $(2 e)$ or $\left(1^{2 e}\right)$. The latter case only occurs in $(\mathrm{d})$ when $(e, r)=(2,1)$.

5.2. Simple endotrivial modules in classical type groups. We will use the fact shown in [11, Thm. 1.3] that any endotrivial module is liftable to a characteristic 0 representation. Therefore, we can investigate endotrivial modules by the ordinary character of their lift. These characters have the following properties:

- they are irreducible modulo $\ell$,

- their values on $\ell$-singular classes are of absolute value 1 ,

- their degree is congruent to \pm 1 modulo $|G|_{\ell}$. 
Theorem 2 is now a consequence of:

Theorem 5.4. Let $G=\operatorname{Sp}_{2 n}(q)$ or $G=\operatorname{Spin}_{2 n+1}(q)$ with $n \geq 2,(n, q) \neq(2,2)$, or $G=\operatorname{Spin}_{2 n}^{ \pm}(q)$ with $n \geq 4$, and $\ell$ a prime for which Sylow $\ell$-subgroups of $G$ are non-cyclic. Let $\rho$ be the character of a nontrivial simple endotrivial $k S$-module, for a central factor group $S$ of $G$. Then we have $G=S=\operatorname{Sp}_{8}(2) \cong \mathrm{O}_{9}(2), \ell=5$, and $\rho=\rho_{\mathcal{S}}$, with $\mathcal{S}=\left(\begin{array}{c}0 \\ 4\end{array}\right)$, is the unipotent character of degree $\rho_{\mathcal{S}}(1)=51$.

Proof. By [11, Thms. 6.7 and 5.2] there do not exist non-trivial simple endotrivial $k S$-modules if either $\ell=2$ or $\ell$ divides $q$. Now let $d=d_{\ell}(q)$. Since Sylow $\ell$ subgroups of $G$ are non-cyclic, the cyclotomic polynomial $\Phi_{d}(q)$ has to divide the group order at least twice. By Theorem 4.1 all non-unipotent characters of $G$ vanish on some $\ell$-singular element of $G$, so cannot come from simple endotrivial modules. The only candidates for endotrivial unipotent characters are those appearing in the conclusion of Theorem 4.2. Of those, we can discard the Steinberg character of $G$, because $G$ contains $\ell$-singular elements with non-trivial unipotent part, and on those classes the value of the Steinberg character is zero.

Now consider the other unipotent characters listed in Theorem 4.2, First assume that $G=\operatorname{Sp}_{2 n}(q)$ or $G=\operatorname{Spin}_{2 n+1}(q)$. Let $\rho=\rho_{\mathcal{S}}$ with $\mathcal{S}$ as in Theorem 4.2(1). So $d$ is odd and $n=2 d+r, 0 \leq r \leq d-1$. Then $\rho$ lies in the principal series and is parameterised by the character of the Weyl group $W\left(B_{n}\right)$ labelled by the pair of partitions $\left(\left(d+r, r+1,1^{d-r-1}\right),-\right) \vdash 2 d+r$. By Proposition 5.2 (a), the corresponding character of the Hecke algebra $\mathcal{H}_{n}$ of type $B_{n}$ is reducible modulo $\ell$ for $r \neq d-1$. Since the decomposition matrix of the Hecke algebra of $W\left(B_{n}\right)$ embeds into that of $G$ (see e.g. [7, Thm. 4.1.14]), this shows that $\rho_{\mathcal{S}}$ is reducible modulo $\ell$, so does not give an example. The same argument applies to the AlvisCurtis dual characters, since Alvis-Curtis duality on the side of Hecke algebras corresponds to tensoring with the sign character. In the case $r=d-1$ we have $\mathcal{S}=\left(\begin{array}{cc}d & 2 d \\ 0\end{array}\right)$ and the corresponding unipotent character does not satisfy the necessary degree congruence by Proposition 5.1 .

So now assume that $d=2 e$ is even and write $n=2 e+r$ with $0 \leq r \leq e-1$. Let $\rho=\rho_{\mathcal{S}}$ as in Theorem 4.2 (2). Again, $\rho$ lies in the principal series and is labelled by the pair of partitions $\left((r+1),\left(e+r, 1^{e-r-1}\right)\right) \vdash 2 e+r$. Hence it is reducible modulo $\ell$ by Proposition 5.2(b) if $e>1$. For $e=1$ we have $r=0, n=2$ and $\mathcal{S}=\left(\begin{array}{cc}0 & 1 \\ 2\end{array}\right)$. Here $\rho_{\mathcal{S}}(1)=q\left(q^{2}+1\right) / 2 \equiv 2 q+1\left(\bmod (q+1)^{2}\right)$ is not congruent to \pm 1 modulo the $\ell$-part of the group order.

For $G=\operatorname{Sp}_{2 n}(q)$ or $G=\operatorname{Spin}_{2 n+1}(q)$ it remains to consider the unipotent characters listed in Theorem 4.2(8) and (9). (Note that the group $\operatorname{Sp}_{4}(2)$ was excluded.) In case (8) we have $q=d=2$, so $\ell=3$. The listed symbol parameterises a unipotent character of degree 7 , its Alvis-Curtis dual has degree 56, both incongruent $\pm 1\left(\bmod 3^{3}\right)$. Finally, in case $(9), \ell \mid\left(q^{2}+1\right)=5$. The second symbol belongs to a character of degree 119, its Alvis-Curtis dual has degree 30464, both incongruent to $\pm 1\left(\bmod 5^{2}\right)$, so this gives no example. But the 51-dimensional simple unipotent module for $\operatorname{Sp}_{8}(2)$ labelled by $\left(\begin{array}{cc}0 & 1 \\ 4\end{array}\right)$ is irreducible and endotrivial in characteristic $\ell=5$. Indeed, the restriction of the corresponding irreducible character to the subgroup $\mathrm{O}_{8}^{+}(2)$ contains the trivial character once and all other constituents are of 5 -defect zero, hence projective modulo 5 . Its Alvis-Curtis dual has degree $13056 \equiv 6$ $\left(\bmod 5^{2}\right)$, so cannot lead to an example. 
Next consider the case that $G=\operatorname{Spin}_{2 n}^{+}(q)$. Let $\rho=\rho_{\mathcal{S}}$ be unipotent with $\mathcal{S}$ as in Theorem $4.2(3)$. So $d$ is odd and $n=2 d+r$ with $0 \leq r \leq d-1$. Then $\rho$ lies in the principal series and is parameterised by the character of the Weyl group $W\left(D_{n}\right)$ labelled by the pair of partitions $(\lambda, \mu)=\left(\left(d+r, r+1,1^{d-r-1}\right),-\right) \vdash 2 d+r$. By Proposition 5.3(a), the corresponding character $\chi_{\lambda, \mu}$ of the Hecke algebra $\mathcal{H}_{n}$ of type $B_{n}$ is reducible modulo $\ell$ for $r \neq d-1$. Since $\lambda \neq \mu, \chi_{\lambda, \mu}$ restricts irreducibly to the Hecke algebra $\mathcal{H}_{n}^{\prime}$ of type $D_{n}$ (of index 2) and thus is also reducible as a character of $\mathcal{H}_{n}^{\prime}$ modulo $\ell$. Using that the decomposition matrix of the Hecke algebra of $W\left(D_{n}\right)$ embeds into that of $G$, this shows that $\rho_{\mathcal{S}}$ is reducible modulo $\ell$, so does not give an example. The same argument applies to the Alvis-Curtis dual characters. In the remaining case $r=d-1$, Proposition 5.1 shows that the corresponding unipotent character does not satisfy the degree congruence.

Now assume that $\rho_{\mathcal{S}}$ is as in Theorem 4.2 (4), so $d=2 e$ is even and $n=2 e+r$ with $0 \leq r \leq e$. Again, $\rho$ lies in the principal series and is labelled by the pair of partitions $\left(\left(e+r, 1^{e-r}\right),(r)\right) \vdash 2 e+r$. By Proposition 5.3(b) the corresponding character of the Hecke algebra of type $B_{n}$ is reducible modulo $\ell$ if $r<e$ and $e>1$, and so as before the same holds for the character of the Hecke subalgebra of type $D_{n}$, whence $\rho_{\mathcal{S}}$ is reducible modulo $\ell$ for $e>1$. Note that $e=1$ is excluded since then $n=2 e=2$. The case when $r=e$ does not satisfy the degree congruence by Proposition 5.1

Similarly, in the case that $G=\operatorname{Spin}_{2 n}^{-}(q)$ the characters of the principal series Hecke algebra $\mathcal{H}_{n-1}\left(q, q^{2}\right)$ labelled by $(\lambda, \mu)$ as in Theorem $4.2(5)$ and $(6)$ are reducible modulo $\ell$ by Proposition 5.3 (c) and (d), so the same holds for the unipotent characters parameterised by these pairs, unless $r=d$. The latter character again does not belong to an endotrivial module by Proposition 5.1. In the exceptional case $(e, r)=(2,1)$ of Proposition 5.3 (d) we have $n=2 e+r=5$ and $\rho_{\mathcal{S}}(1)+1 \equiv 4\left(q^{2}+1\right)$ $\left(\bmod \left(q^{2}+1\right)^{2}\right)$, hence the necessary degree congruence is not satisfied for $\rho_{\mathcal{S}}$.

Finally, the character listed in Theorem 4.2(10) takes value 2 on some $\ell$-singular classes, by the final statement in that result, so again cannot be endotrivial.

The endotrivial characters of $\operatorname{Sp}_{4}(2) \cong \mathfrak{S}_{6}$ and its covers have been described in [11, Thm. 4.9].

As pointed out before, the simple endotrivial modules of linear and unitary groups have already been determined in [10, Thm. 3.10 and 4.5].

\section{ZERoEs of CHARACTERS OF QUASI-SIMPLE GROUPS}

Together with our previous work [10,11] with C. Lassueur and E. Schulte our results allow us to guarantee the existence of zeroes of characters of quasi-simple groups on $\ell$-singular elements once the $\ell$-rank is at least 3 , as claimed in Theorem 1 which we restate:

Theorem 6.1. Let $\ell>2$ be a prime and $G$ a finite quasi-simple group of $\ell$-rank at least 3. Then for any non-trivial character $\chi \in \operatorname{Irr}(G)$ there exists an $\ell$-singular element $g \in G$ with $\chi(g)=0$, unless one of:

(1) $G$ is of Lie type in characteristic $\ell$;

(2) $\ell=5, G=\mathrm{L}_{5}(q)$ with $5 \|(q-1)$ and $\chi$ is unipotent of degree $\chi(1)=q^{2} \Phi_{5}$;

(3) $\ell=5, G=\mathrm{U}_{5}(q)$ with $5 \|(q+1)$ and $\chi$ is unipotent of degree $\chi(1)=q^{2} \Phi_{10}$;

(4) $\ell=5, G=L y$ and $\chi(1) \in\{48174,11834746\}$; or 
(5) $G=E_{8}(q)$ with $q$ odd, $d_{\ell}(q)=4$ and $\chi$ is the semisimple character with label $((0),(8))$ in the unique Lusztig-series of type $D_{8}$.

Proof. For $G$ a covering group of a sporadic simple group, an easy check of the known character tables shows that only case (4) arises. For alternating groups and their covering groups the claim is contained in the proof of [11, Prop. 4.2 and Thm. 4.5]. If $G$ is a covering group of a special linear or unitary group then [10, Thm. 1.3] shows that only the cases in (1), (2) and (3) can arise.

For the remaining groups of Lie type first note that the Steinberg character vanishes on the product of any $\ell$-element with a unipotent element in its centraliser, so it can be discarded from our discussion. We now first consider groups $G$ of exceptional Lie type. Since the $\ell$-rank of $G$ is at least 3 , it must be of type $F_{4}$, $E_{6},{ }^{2} E_{6}, E_{7}$ or $E_{8}$. For these it is shown in the proof of [11, Thm. 6.11] that their irreducible characters $\chi$ vanish on some $\ell$-singular element unless either $\chi$ is unipotent or $\ell \mid\left(q^{2}+1\right)$ in $G=E_{8}(q)$ and $\chi$ lies in the Lusztig series of an isolated element with centraliser $D_{8}(q)$. We deal with these cases in turn.

We begin with $G=E_{8}(q)$. Since $G$ has $\ell$-rank at least 3 we must have $d=d_{\ell}(q) \in$ $\{1,2,3,4,6\}$. First assume that $d=1$. There exist maximal tori of orders divisible by $\Phi_{1} \Phi_{7}, \Phi_{1} \Phi_{9}$ and $\Phi_{1} \Phi_{14}$, hence $G$ contains semisimple $\ell$-singular elements of order divisible by a Zsigmondy prime divisor $r$ of $\Phi_{7}, \Phi_{9}$ and $\Phi_{14}$. All non-trivial unipotent characters apart from $\phi_{8,1}, \phi_{8,91}$ and the Steinberg character are of defect zero for one of these three primes, hence vanish on any $r$-singular element. For the remaining two characters, direct calculation with the character formula shows that they vanish on elements of order $\Phi_{1} \Phi_{4}$. Very similar considerations apply to the remaining cases $d \in\{2,3,4,6\}$. If $d=4$ and $\chi$ lies in the Lusztig series of an isolated element with centraliser $D_{8}(q)$, the same argument using Zsigmondy prime divisors of $\Phi_{5}, \Phi_{8}, \Phi_{10}$ and $\Phi_{12}$ only leaves four characters in that series, those with label $\left(\begin{array}{l}0 \\ 8\end{array}\right)$ and $\left(\begin{array}{l}01 \\ 18\end{array}\right)$ and their duals. Three of them are zero on certain products of an $\ell$ element with a unipotent element.

The arguments for the unipotent characters of the remaining exceptional groups are similar and easier.

Finally, let us suppose that $G$ is of classical Lie type. By Theorem 4.1 the claim holds if $\chi$ is not unipotent. For unipotent characters $\chi$, the claim follows from Theorem 4.2, since all exceptions listed there have $\ell$-rank two, including those in cases (4) and (5) with $n=3 e$, respectively, $n=3 d$.

Remark 6.2. (a) Let $p$ be a prime and $f \geq 1$. Then $G=\mathrm{SL}_{2}\left(p^{f}\right)$ has $p$-rank $f$, but its irreducible characters of degree $q \pm 1$ do not vanish on any $p$-singular elements of $G$. Similarly, there exist such characters for $\mathrm{SL}_{3}\left(p^{f}\right), \mathrm{SU}_{3}\left(p^{f}\right)$ and ${ }^{2} G_{3}\left(3^{2 f+1}\right)$. This shows that Case (1) in Theorem 6.1 is a true exception. Also, Ly has characters of degrees 48174 and 11834746 which do not vanish on 5 -singular elements. (It is well known that the Lyons group behaves like a characteristic 5 group in many respects; see e.g. [14.) It can be checked that the unipotent characters of $\mathrm{L}_{5}(q)$ and $\mathrm{U}_{5}(q)$ listed in Cases (2) and (3) are also true exceptions.

(b) We expect the exceptions in Case (1) to only occur for groups of Lie type in characteristic $\ell$ of small Lie rank.

(c) The results of [11, [10] and of the present paper show that even for $\ell$-rank 2 there exist only relatively few irreducible characters of quasi-simple groups not vanishing on some $\ell$-singular element. Nevertheless we refrain from attempting to give an explicit list in that case. 


\section{An application to small 1-PIMs}

Our results so far may also be used in order to investigate the first Cartan invariant of finite simple classical groups. In their recent paper [9], Koshitani, Külshammer and Sambale raised a question to understand simple groups with noncyclic Sylow $\ell$-subgroup for which the first Cartan invariant $c_{11}$ equals 2.

Theorem 7.1. Let $G$ be a finite simple group of classical Lie type $B_{n}, C_{n}, D_{n}$ or ${ }^{2} D_{n}$. Let $\ell>2$ be a prime for which Sylow $\ell$-subgroups of $G$ are non-cyclic. Then the $\ell$-modular projective cover of the trivial character of $G$ has at least three ordinary constituents. In particular, $c_{11} \geq 3$.

Proof. For $\ell$ the defining characteristic of $G$, this was already shown in [9]. Now assume that $\ell \neq 2$ is different from the defining characteristic of $G$. Assume that the character of the $\ell$-modular projective cover of the trivial $G$ module has the form $1_{G}+\chi$, with $\chi \in \operatorname{Irr}(G)$. Since projective characters vanish on all $\ell$-singular elements, this implies that $\chi(g)=-1$ on all elements $g \in G$ of order divisible by $\ell$.

We now use our results on zeroes of characters. By Theorem 4.1 any nonunipotent character of $G$ vanishes on some $\ell$-singular element. (Again note that $\mathrm{Sp}_{4}(2) \cong \mathfrak{S}_{6}$ is not simple.) So $\chi$ must be unipotent. In this case, Theorem 4.2 again shows that most unipotent characters also vanish on some $\ell$-singular element. It remains to consider the listed exceptions.

The trivial character never has value -1 . To rule out the Steinberg character we use that there is always a non-regular element $s \in G$ of order $\ell$, hence there is a unipotent element $u \neq 1$ in the centraliser of $s$. The Steinberg character vanishes on the non-semisimple element su. For the small cases listed in 4.2 (7) to (10) and their Alvis-Curtis duals we just check that there is always an $\ell$-singular regular semisimple element on which the character has value $\neq-1$.

It remains to consider the unipotent characters corresponding to symbols in Theorem 4.2(1) to (6) and their duals. We have mentioned that the values of these characters on $\ell$-singular regular semisimple classes are \pm 1 . We give for each case a pair of parameters of maximal tori on which character values have opposite signs. This is easy to compute with the Murnaghan-Nakayama formula (Theorem 3.3) using the definitions of the signs occurring there. It turns out that in each case the same pair of tori works for the symbol and its dual. The dual symbols and the pairs of tori are as follows:

(1) $\mathcal{S}^{\vee}=(\underset{d+r}{\stackrel{d+r}{\backslash 0 \backslash d} \cup 2 d})$, tori $((d),(d+r))$ and $((2 d),(r))$,

(2) $\mathcal{S}^{\vee}=\left(\frac{\overline{e+r} \backslash 0 \cup 2 e}{e+r} \backslash e\right)$, tori $((2 e),(r))$ and $((e+r),(e))$,

(3) $\mathcal{S}^{\vee}=\left(\frac{\frac{d+r-1}{d+r \backslash 0 \backslash d \cup 2 d}}{d+}\right)$, tori $((d, d+r),-)$ (or $((d, d-2),(1,1)$ if $r=0)$ and $((2 d, r),-)$ (or $((2 d),(1,1)$ if $r=2)$,

(4) $\mathcal{S}^{\vee}=\left(\frac{e+r-1}{e+r} \backslash e(2 e)\right.$, tori $((2 e),-)$ and $((e-1),(e, 1))$ if $r=0$ (use $((1),(3,2))$ for $d=n \overline{=6})$ and in case $r>0$ tori $(-,(e+r, e))$ and $((2 e, r),-)($ if $r \neq 2)$ or $((2 e),(1,1))$ (if $r=2)$,

(5) $\mathcal{S}^{\vee}=(\underline{d+r-\overline{1} \backslash 0 \backslash d \cup 2 d})$, tori $((d),(d+r))$ and $((2 d),(r))$,

(6) $\mathcal{S}^{\vee}=\left(\frac{\overline{e+r} \backslash 0 \cup 2 e}{e+r-1 \backslash e}\right)$, tori $((e+r),(e))$ and $((2 e),(r))$.

The only cases which are not covered by this argument because one of the given tori has no regular element are: $\operatorname{Sp}_{4}(2)$ with $d=2(\ell=3)$ and $\operatorname{Sp}_{8}(2)$ with $d=4$ 
$(\ell=5)$. The first group is not simple, and for the second the claim can be checked from its character table.

\section{ACKNOWLEDGEMENTS}

The authors thank Andrew Mathas for useful explanations concerning the notation and results of 8 . We also thank the anonymous referee for his helpful comments.

\section{REFERENCES}

[1] T. Asai, Unipotent class functions of split special orthogonal groups $\mathrm{SO}_{2 n}^{+}$over finite fields, Comm. Algebra 12 (1984), no. 5-6, 517-615, DOI 10.1080/00927878408823017. MR735137 (86a:20044)

[2] T. Asai, The unipotent class functions on the symplectic groups and the odd orthogonal groups over finite fields, Comm. Algebra 12 (1984), no. 5-6, 617-645, DOI 10.1080/00927878408823018. MR735138 (86a:20045)

[3] T. Asai, The unipotent class functions of nonsplit finite special orthogonal groups, Comm. Algebra 13 (1985), no. 4, 845-924, DOI 10.1080/00927878508823197. MR776867 (86k:20039)

[4] R. W. Carter, Finite groups of Lie type: Conjugacy classes and complex characters; A WileyInterscience Publication, Pure and Applied Mathematics (New York), John Wiley \& Sons, Inc., New York, 1985. MR794307(87d:20060)

[5] F. Digne and J. Michel, Representations of finite groups of Lie type, London Mathematical Society Student Texts, vol. 21, Cambridge University Press, Cambridge, 1991. MR.1118841 (92g:20063)

[6] P. Fong and B. Srinivasan, Generalized Harish-Chandra theory for unipotent characters of finite classical groups, J. Algebra 104 (1986), no. 2, 301-309, DOI 10.1016/00218693(86)90217-6. MR866777 (88a:20057)

[7] M. Geck and N. Jacon, Representations of Hecke algebras at roots of unity, Algebra and Applications, vol. 15, Springer-Verlag London, Ltd., London, 2011. MR2799052 (2012d:20010)

[8] G. James and A. Mathas, The Jantzen sum formula for cyclotomic $q$-Schur algebras, Trans. Amer. Math. Soc. 352 (2000), no. 11, 5381-5404, DOI 10.1090/S0002-9947-00-02492-2. MR.1665333 (2001b:16017)

[9] S. Koshitani, B. Külshammer, and B. Sambale, On Loewy lengths of blocks, Math. Proc. Cambridge Philos. Soc. 156 (2014), no. 3, 555-570, DOI 10.1017/S0305004114000103. MR3181640

[10] C. Lassueur and G. Malle, Simple endotrivial modules for linear, unitary and exceptional groups, Math. Z. 280 (2015), no. 3-4, 1047-1074, DOI 10.1007/s00209-015-1465-0. MR 3369366

[11] C. Lassueur, G. Malle, E. Schulte, Simple endotrivial modules for quasi-simple groups, J. Reine Angew. Math. DOI: 10.1515/crelle-2013-0100.

[12] G. Lusztig, Characters of reductive groups over a finite field, Annals of Mathematics Studies, vol. 107, Princeton University Press, Princeton, NJ, 1984. MR742472 (86j:20038)

[13] G. Malle and D. Testerman, Linear Algebraic Groups and Finite Groups of Lie Type, Cambridge Studies in Advanced Mathematics, vol. 133, Cambridge University Press, Cambridge, 2011. MR2850737(2012i:20058)

[14] C. Parker and P. Rowley, A characteristic 5 identification of the Lyons group, J. London Math. Soc. (2) 69 (2004), no. 1, 128-140, DOI 10.1112/S0024610703004848. MR2025331 (2004j:20027)

[15] G. Pfeiffer, Character tables of Weyl groups in GAP, Bayreuth. Math. Schr. 47 (1994), 165222. MR1285208 (95d:20027)

Lehrstuhl D für Mathematik, RWTH Aachen, Pontdriesch 14/16, 52062 Aachen, GerMANY.

E-mail address: Frank.Luebeck@math.rwth-aachen.de

FB Mathematik, TU Kaiserslautern, Postfach 3049, 67653 Kaiserslautern, Germany. E-mail address: malle@mathematik.uni-kl.de 